\title{
Biocompatible fluorescent silicon nanocrystals for single-molecule tracking and fluorescence imaging
}

\author{
Hirohito Nishimura, ${ }^{1,2}$ Ken Ritchie, ${ }^{4}$ Rinshi S. Kasai, ${ }^{1}$ Miki Goto, ${ }^{1}$ Nobuhiro Morone, ${ }^{1}$ Hiroyuki Sugimura, ${ }^{3}$ \\ Koichiro Tanaka, ${ }^{1}$ Ichiro Sase, ${ }^{5}$ Akihiko Yoshimura, ${ }^{6}$ Yoshitaro Nakano, ${ }^{5}$ Takahiro K. Fujiwara, ${ }^{1}$ and Akihiro Kusumi ${ }^{1,2}$ \\ Institute for Integrated Cell-Material Sciences, ${ }^{2}$ Institute for Frontier Medical Sciences, and ${ }^{3}$ Department of Materials Science and Engineering, Kyoto University, \\ Kyoto 606-8501, Japan \\ ${ }^{4}$ Department of Physics, Purdue University, West Lafayette, IN 47907 \\ ${ }^{5}$ Instruments Company, Nikon Corporation, Yokohama 244-8533, Japan \\ 'Department of Microbiology and Immunology, Keio University School of Medicine, Tokyo 160-8582, Japan
}

\section{Introduction}

Fluorescence microscopy is one of the most important tools for cell-biological and biomedical research as well as clinical diagnostics (Torres et al., 2008; Toomre and Bewersdorf, 2010; Yan et al., 2011; Germain et al., 2012). However, three major problems with the presently available fluorescent probes still often appear in fluorescence microscopy: (1) photobleaching, (2) blinking (Dickson et al., 1997; Yao et al., 2005), which almost all of the presently available probes exhibit, and (3) the large size of GFP and quantum dots (QDs). Extensive efforts have been made to produce QDs and organic fluorescent molecules to alleviate these problems (Buschmann et al., 2003; Howarth et al., 2008; Muro et al., 2010; Altman et al., 2012a,b; Liu et al., 2012), but their applicability to living cells, particularly for singlemolecule imaging using living cells (Cognet et al., 2006; Smith

H. Nishimura and K. Ritchie contributed equally to this paper.

Correspondence to Akihiro Kusumi: akusumi@frontier.kyoto-u.ac.jp

Abbreviations used in this paper: 3-MPTS, 3-mercaptopropyltrisilane; CCD, chargecoupled device; CEF, chick embryonic fibroblast; CSD, cumulative SD; DLS, dynamic light scattering; FCS, fluorescence correlation spectroscopy; FTIR, Fourier transform infrared; hTfR, human TfR; NRK, normal rat kidney; QD, quantum dot; $\mathrm{SD}$, square displacement; $\mathrm{SiNC}$, silicon nanocrystal; sulfo-EMCS, $N$ - $(\varepsilon$-maleimi docaproyloxy)sulfosuccinimide ester; TEM, transmission EM; Tf, transferrin; TfR, Tf receptor; TIRF, total internal reflection fluorescence.

Single SiNCs neither blinked nor photobleached during a 300-min overall period observed at video rate. Single receptor molecules in the plasma membrane of living cells (using transferrin receptor) were imaged for $\geq 10$ times longer than with other probes, making it possible for the first time to observe the internalization process of receptor molecules at the single-molecule level. Spatial variations of molecular diffusivity in the scale of $1-2 \mu \mathrm{m}$, i.e., a higher level of domain mosaicism in the plasma membrane, were revealed.

and Nie, 2010; Kasai et al., 2011), has been severely limited because the problems of photobleaching, blinking, and crosslinking target molecules are more critical in single-molecule imaging in living cells (Ha and Tinnefeld, 2012).

Physicists and chemists have known for almost two decades that silicon nanocrystals ( $\mathrm{SiNCs}$ ) as small as $1-5 \mathrm{~nm}$ in diameter are fluorescent (Canham, 1990; Yamani et al., 1998; Credo et al., 1999; Akcakir et al., 2000), with an emission spectrum that varies with the particle size, from blue from the smallest to red from the largest (Belomoin et al., 2002; Zhou et al., 2003; Sychugov et al., 2005a), do not easily photobleach (Gelloz et al., 2003; Warner et al., 2005), and could be made water soluble (Li et al., 2004; Li and Ruckenstein, 2004; Warner et al., 2005) and targeted to biological molecules (Wang et al., 2004; Erogbogbo et al., 2008, 2011a,b). Recently, a possibility of greatly improving the quantum efficiency of SiNCs has been proposed (de Boer et al., 2010).

(c) 2013 Nishimura et al. This article is distributed under the terms of an AttributionNoncommercial-Share Alike-No Mirror Sites license for the first six months after the publication date (see http://www.rupress.org/terms). After six months it is available under a Creative Commons License (Attribution-Noncommercial-Share Alike 3.0 Unported license, as described at http://creativecommons.org/licenses/by-nc-sa/3.0/). 


\section{A Chemical Etching}
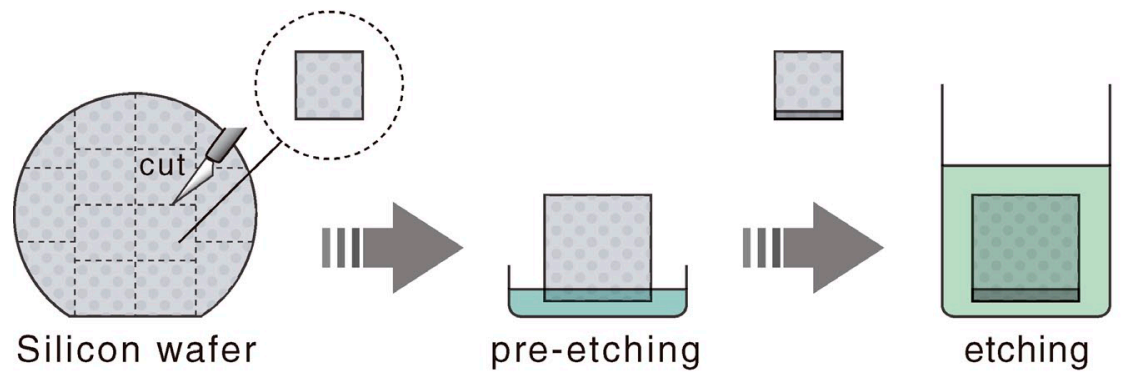

\section{Liberation and Purification}
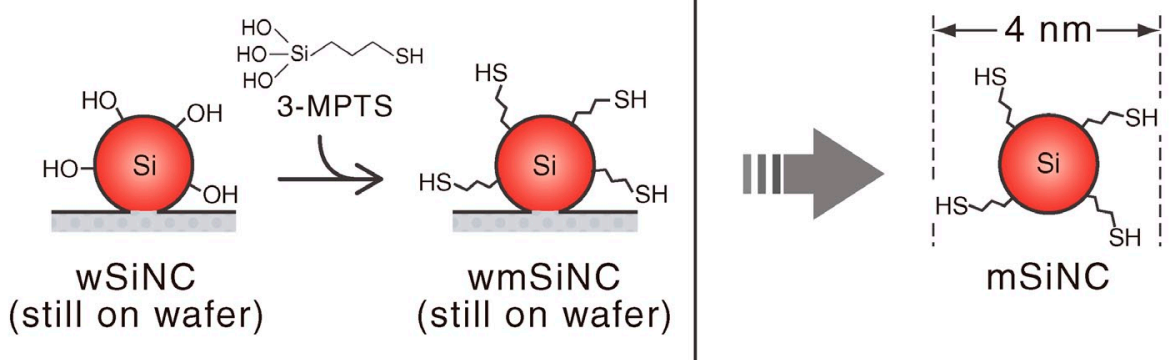

Figure 1. Preparation of hydrophilic mercaptosilane-coated SiNC and $\mathrm{mSiNC}$. (A-C) Schemes for chemical etching of the silicon wafer to produce fluorescent $\mathrm{SiNCs}$ on the wafer surface (wSiNCs; A), mercaptosilane coating of the wafer (wmSiNCs; B), and removing nanocrystals by mechanical scraping, to produce mercaptosilane-coated SiNCs dispersed in water (mSiNCs; C). These mSiNCs were then purified by Superdex 75 gel filtration HPLC.

Therefore, we considered SiNCs as candidates that could simultaneously and fundamentally solve all of the three major problems of present fluorescent probes and in addition could make ideal single fluorescent molecule-imaging probes. However, despite all the aforementioned developmental efforts, the application of SiNCs to cell-biological and biomedical research has been extremely limited (Choi et al., 2008; Erogbogbo et al., 2008, 2011a,b). No serious application has been made for single fluorescent molecule imaging and tracking (Akcakir et al., 2000). This is largely caused by the following three difficulties: (1) in producing SiNCs, (2) in producing particle populations with uniform size and properties in aqueous media (particularly redemitting SiNCs), and (3) in conjugating them to biomolecules at a 1:1 mol ratio. Furthermore, each of these problems as well as issues of purification, monodispersibility, size distribution, and determinations of extinction coefficient, quantum yield, the fraction of actually fluorescent nanocrystals, and photobleaching/ blinking time has been addressed separately, and previously, these problems have never been simultaneously solved for a single type of SiNCs. Therefore, specific objectives of the present research are threefold: (1) to develop SiNCs that solve all of these three problems (for two-color imaging with GFP in future studies, we focused on red-emitting SiNCs), (2) to characterize the developed SiNCs at the level of single particles, which is rather new, and (3) to apply the developed SiNCs to long-term single-molecule tracking of receptor molecules on the living cell surface.

\section{Results}

\section{Preparing mercaptosilane-coated SiNCs with an 4-nm hydrodynamic diameter}

Inspired by the work of Yamani et al. (1998), Akcakir et al. (2000), and Belomoin et al. (2002), we developed an easier protocol that specifically generates red-emitting SiNCs (see Materials and methods subsections Preparation of SiNC on the wafer through Recovery and purification of mSiNC; Fig. 1, A-C). This method employs the simple chemical etching of silicon wafers (Fig. $1 \mathrm{~A}$ ), rather than the electrochemical etching used previously. Then, the SiNCs, still attached to the wafer (wSiNCs), were coated by conjugating 3-mercaptopropyltrihydroxysilane (3-MPTS) to the hydroxyl (silanol) groups on the wSiNC surface (wmSiNCs; Fig. 1 B). Coating was confirmed by Fourier transform infrared (FTIR) spectroscopy (Fig. 2 A, top). The fluorescence spectrum of $\mathrm{wSiNC}$ and $\mathrm{wmSiNC}$ are shown in Fig. 2 B. The fluorescence spectrum changed slightly after hydrophilic coating. The SiNCs were then liberated from the polished side of the wafer by mechanical scraping into water. This suspension was centrifuged briefly to remove the large debris and then was purified by gel filtration HPLC (mSiNCs; Fig. 1 C). FTIR spectroscopy confirmed that the 3-mercaptopropyltrisilane (3-MPTS) remained on the $\mathrm{mSiNC}$ surface after this procedure (Fig. 2 A, bottom).

In the gel filtration HPLC using a Superdex 75 column, the purified mSiNCs eluted in a sharp band between cobalamin 
A

C

D
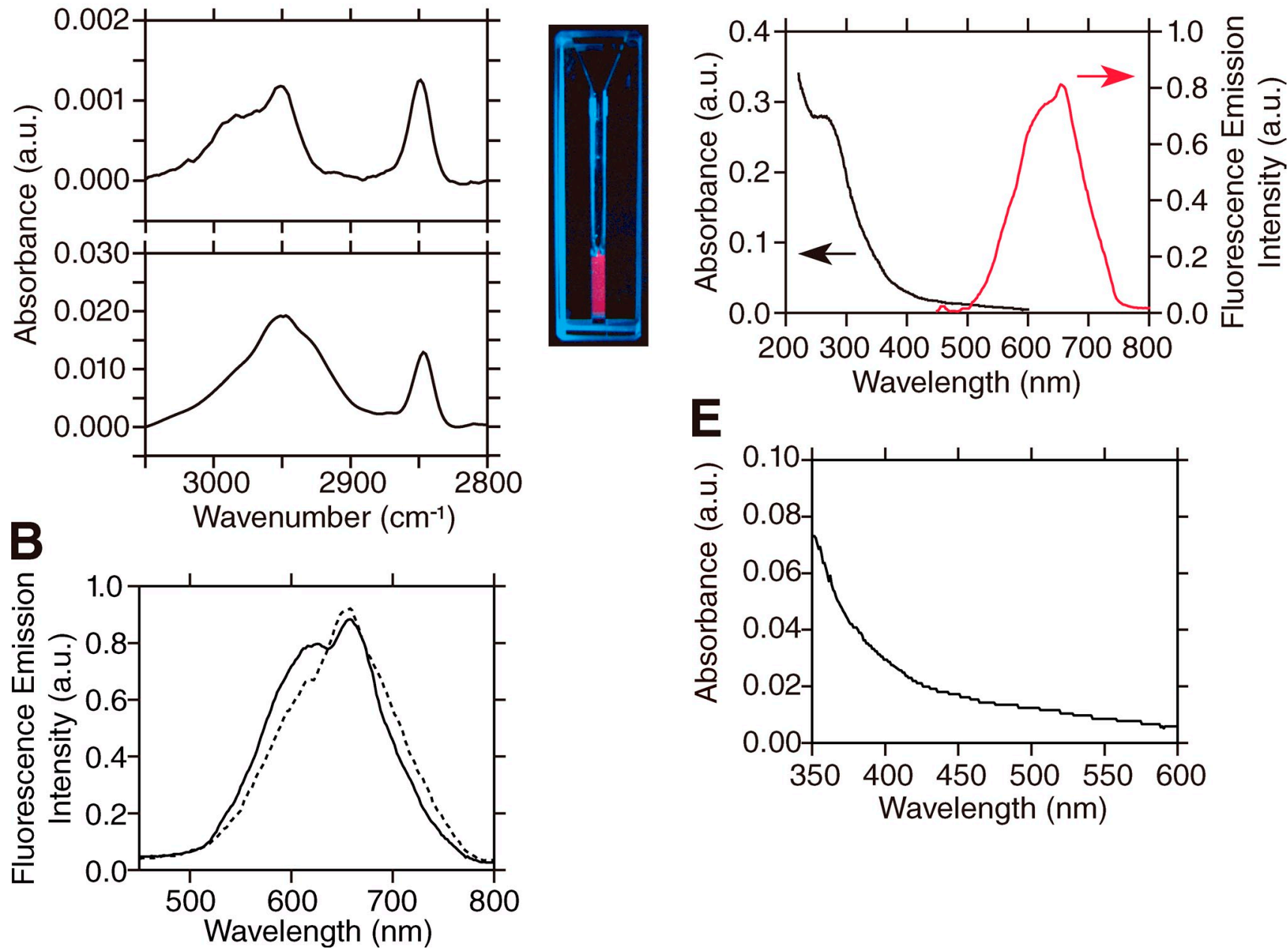

Figure 2. Fluorescence and FTIR spectra of wmSiNC and purified mSiNC. (A-E) Typical spectra among $3(A), 9(B)$, and 17 (D and E) entirely independent experiments are shown. (A) FTIR spectra of wmSiNCs (top) and $m \mathrm{SiNCs}$ after gel filtration HPLC (bottom), with peaks corresponding to alkyl C-H $\mathrm{H}_{\mathrm{x}}$ bonds at 2,949 and 2,847 $\mathrm{cm}^{-1}$, indicating the 3-MPTS binding to SiNCs. (B) Fluorescence spectra of wSiNCs (dashed line) and wmSiNCs (solid line) excited at $405 \mathrm{~nm}$. (C) Fluorescence image of a cuvette containing mSiNCs, excited with a UV lamp. (D) Absorption (black; y axis on the left, see the black arrow) and fluorescence (red, excitation at $405 \mathrm{~nm}$; y axis on the right, see the red arrow) spectra for mSiNCs after Superdex 75 gel filtration HPLC in $50 \mathrm{mM}$ phosphate buffer, pH 7.0. (E) The expanded absorption spectrum of mSiNC (350-600 nm). a.u., arbitrary unit.

vitamin B12 (hydrodynamic diameter $\sim 1.66 \mathrm{~nm}$; Colton et al., 1971) and RNase A (hydrodynamic diameter $\sim 4.24 \mathrm{~nm}$; O'Connor et al., 2007; Fig. 3 A). This result strongly suggests that mSiNCs act like water-soluble molecules with a uniform diameter, an important property for a biomedical probe. Fig. $3 \mathrm{~B}$ shows the elution pattern of the gel filtration HPLC of the mSiNC solution that also contained Qdot655 ITK carboxyl QDs (Qdot655; Invitrogen) and GFP. To avoid GFP dimerization, we used GFP with the A206K mutation (Zacharias et al., 2002). Qdot655 is widely used as a canonical QD, and it was used as the standard QD throughout the present research. Fig. 3 B shows that the mSiNCs are smaller than GFP, which, in turn, is smaller than Qdot655. In the subsequent examinations of mSiNCs, mSiNCs eluted from the Superdex 75 column in a peak at $\sim 38$ min were always used.

Using dynamic light scattering (DLS; see DLS measurement of the $\mathrm{mSiNC}$ size in Materials and methods; Fig. $3 \mathrm{C}$ ), the hydrodynamic diameter of the mSiNCs was determined to be $4.1 \pm 0.14 \mathrm{~nm}$ with a polydispersity index of $0.14 \pm 0.028$ (mean \pm SD throughout this paper, where SD represents the SD of the mean value for $n$ independent experiments; $n=3$ here), indicating a sharp distribution of the particle size (Fig. $3 \mathrm{C}$, curve). It is concluded that, after Superdex 75 gel filtration HPLC, the particle size was uniform, and the contamination of larger particles, mSiNC dimers, and greater clusters was very limited.

The $\mathrm{mSiNC}$ in aqueous buffer was further characterized by fluorescence correlation spectroscopy (FCS; see FCS and determination of the mSiNC size based on the FCS measurements in Materials and methods). Typical plots of the autocorrelation function versus time for $\mathrm{mSiNC}$, GFP, Qdot655, and rhodamine $6 \mathrm{G}$ are shown in Fig. $3 \mathrm{D}$. The hydrodynamic diameter of $\mathrm{mSiNC}$ was determined to be $4.1 \pm 0.2 \mathrm{~nm}(n=6$; see FCS and determination of the mSiNC size based on the FCS measurements), which was smaller than those of GFP (5.5 \pm $0.1 \mathrm{~nm} ; n=4)$ and Qdot655 (19.4 $\pm 0.5 \mathrm{~nm} ; n=4)$, consistent with the gel filtration HPLC result. 

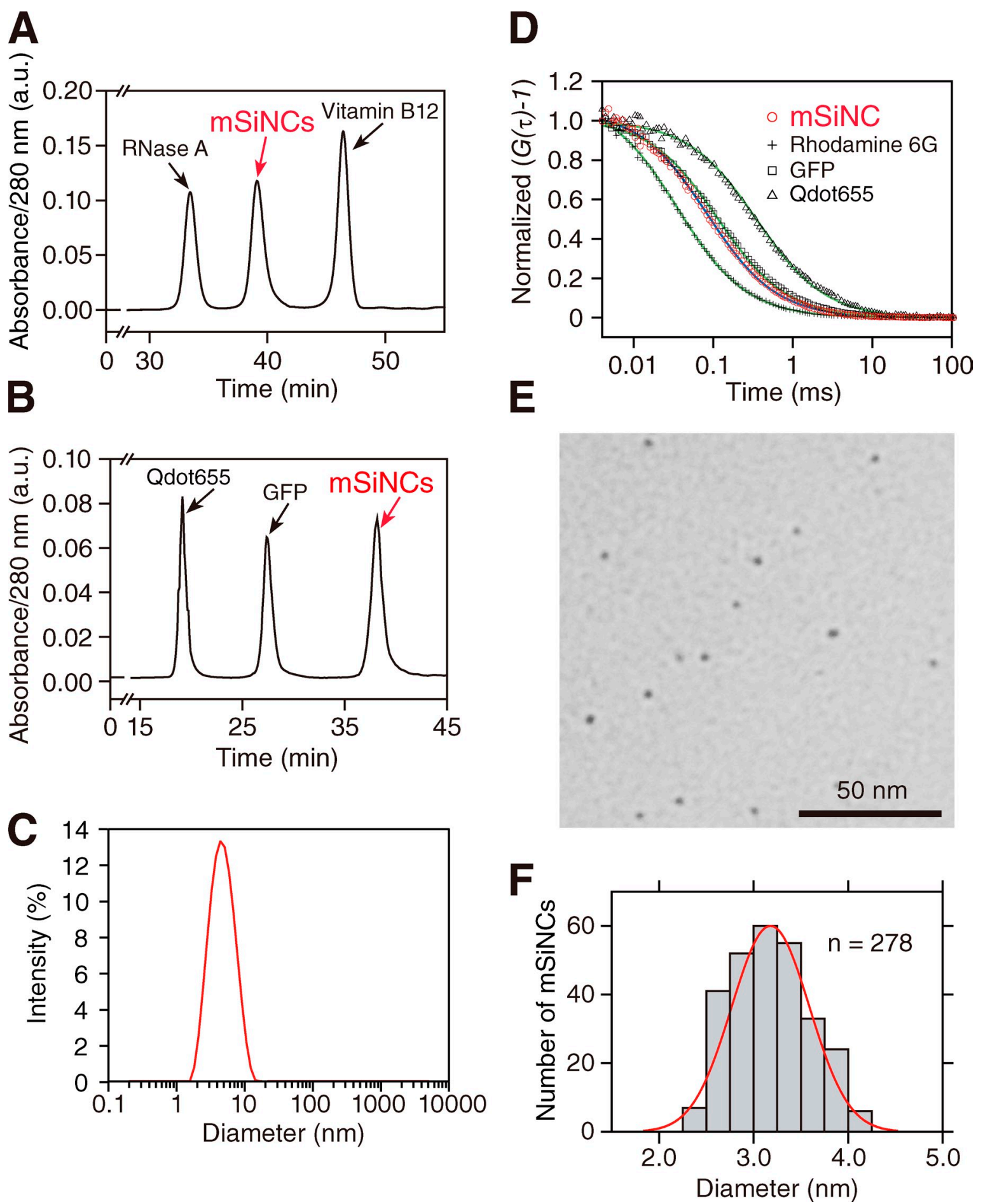

Figure 3. Characterization of purified mSiNC. Typical results among 7 (A), 5 (B), $3(C)$, and 6 (D) entirely independent experiments are shown. (A) Superdex 75 gel filtration HPLC elution pattern of $\mathrm{mSiNC}$ loaded with the sizing controls of vitamin B12 and RNase A. (B) Superdex 75 gel filtration HPLC pattern of Qdot655 (Qdot655 ITK carboxyl QDs), GFP, and mSiNC. The mSiNC is much smaller than GFP and Qdot655. Data in early time periods were deleted in $A$ and $B$ because no elvent peaks were detectable during these early periods. (C) A typical DLS of purified mSiNC, shown as the scattered light intensity versus particle diameter (see DLS measurement of the mSiNC size). (D) Fluorescence autocorrelation curve $G(\tau)$ obtained from FCS measurements. The solid lines represent the fits by Eq. 1 in FCS and determination of the mSiNC size based on the FCS measurements. (E) A representative transmission electron micrograph of $m S i N C s$ after Superdex 75 gel filtration HPLC. (F) The size distribution of $m S i N C s$ obtained from the transmission electron micrograph. The red solid line represents the fit by the Gaussian function $(3.2 \pm 0.4 \mathrm{~nm})$. The results of three totally independent experiments are summarized to obtain this distribution ( $n=278$ particles). a.u., arbitrary unit.

Transmission EM (TEM) images of the mSiNCs recovered from the gel filtration peak (Fig. $3 \mathrm{E}$ ) provided the $\mathrm{mSiNC}$ diameter distribution (Fig. 3 F), which can be fitted by a Gaussian function $(3.2 \pm 0.4 \mathrm{~nm} ; n=278$ particles). Because this diameter represents that of the $\mathrm{mSiNC}$ core without including the surface coating, and in addition, the hydrodynamic diameter represents 
that of the hydrodynamic slipping layer around $\mathrm{mSiNC}$, the smaller TEM diameter is consistent with the 4.1-nm hydrodynamic diameter determined by DLS and FCS.

A fluorescence image of a cuvette containing mSiNCs purified by gel filtration HPLC, excited with a UV lamp, is shown in Fig. 2 C. The absorption and fluorescence spectra of the purified $\mathrm{mSiNCs}$ are shown in Fig. 2 (D and E). The peak of the fluorescence spectrum is $\sim 655 \mathrm{~nm}$, and its width is broader (full width at half maximum $=\sim 100 \mathrm{~nm}$ ) than that for the QDs by a factor of 2-3, consistent with a previous finding that even a uniform population of blue-emitting SiNCs of $1.4 \pm 0.3 \mathrm{~nm}$ in diameter exhibited the fluorescence spectrum with a full width at half maximum of $\sim 80 \mathrm{~nm}$ (Warner et al., 2005).

Storing the mSiNCs in dehydrated benzene at $4{ }^{\circ} \mathrm{C}$ for $60 \mathrm{~d}$ did not affect either their fluorescence spectrum or fluorescence intensity (Fig. S1), as also reported for hydrophobic SiNC in toluene (Li et al., 2004). However, in aqueous solutions, the fluorescence intensity was reduced much faster. The fluorescence intensity of $\mathrm{mSiNC}$ conjugated with a single molecule of transferrin (Tf; $\mathrm{mSiNC}$-Tf) decreases with an exponential decay time of $42 \pm 2.8 \mathrm{~h}$ in $\mathrm{PBS}$ at $37^{\circ} \mathrm{C}(n=4$; the fitting error at the $68.3 \%$ confidence limit is given; see Long-term storage mSiNCs and the preparation and observation of $\mathrm{mSiNC}$-Tf in aqueous solutions during a day in Materials and methods; Fig. S2).

\section{Each individual mSiNC neither blinks} nor photobleaches

To determine whether each mSiNC blinks or photobleaches, purified mSiNCs were adsorbed and immobilized on polylysinecoated coverslips and were imaged with a home-built objective lens-type total internal reflection fluorescence (TIRF) microscope (a 440-nm laser line with $0.34 \mu \mathrm{W} / \mu \mathrm{m}^{2}$ on the sample plane; Fig. 4 A; Koyama-Honda et al., 2005). The distribution of the fluorescence intensities of individual mSiNCs (log-normal fitting; Mutch et al., 2007; Fig. 4 B, red line) is consistent with the presence of a population of single fluorescent particles (Fig. 4 B, top). Meanwhile, the fluorescence intensities of single individual Qdot655 particles exhibited much greater variations (Fig. 4 B, bottom).

A representative plot of the fluorescence intensity versus time of a single $\mathrm{mSiNC}$ at a $33-\mathrm{ms}$ (video rate) resolution is shown in Fig. $4 \mathrm{C}$ (top) and should be compared with that of a CdSe-QD (Qdot655 here) observed under exactly the same illumination and detection conditions (Fig. 4 C, bottom). Qdot655 exhibited frequent blinking (for the nature of the blinking and its mechanism for QDs, see Chen et al. [2008], Mahler et al. [2008], and Wang et al. [2009]) as well as gradual photobleaching (Fig. $4 \mathrm{C}$ and Fig. S3 B), consistent with previous observations (van Sark et al., 2002). None of the $30 \mathrm{mSiNCs}$ observed under the same conditions blinked or photobleached (during the total period of $300 \mathrm{~min}$ ), although Sychugov et al. (2005b) reported the blinking of SiNCs. Under the conditions in which similar signal-to-noise ratios are attained for single fluorescent spots, single molecules of GFP or the organic dye Cy3 photobleached within $\sim 3$ s (unpublished data). Namely, by using mSiNCs as fluorescent probes, the observation time of single molecules could be lengthened by a factor of 6,000 or more (without a single blinking event), eliminating the two major roadblocks for single-molecule tracking.

The average fluorescence signal intensity for a single $\mathrm{mSiNC}$ particle, as determined by the log-normal fitting of the distribution of signal intensities of single mSiNCs (Fig. S4 A) is increased linearly at least $\leq 0.68 \mu \mathrm{W} / \mu \mathrm{m}^{2}$ on the sample plane (Fig. S4 B), twice the standard laser power used in this work. No photobleaching and blinking were observed even at $0.68 \mu \mathrm{W} / \mu \mathrm{m}^{2}$.

We noted that many QDs suddenly started emitting fluorescence sometime after irradiation with the excitation light, consistent with a previous study (for example, see Li-Shishido et al., 2006). This result suggests that significant fractions of Qdot655 and mSiNCs might be nonfluorescent at a given time. In fact, Credo et al. (1999) reported that only $2.8 \%$ of SiNCs are fluorescent.

Therefore, we examined the fractions of nonfluorescent mSiNCs using Alexa Fluor 488-conjugated mSiNC (see Preparation of Alexa Fluor 488-labeled mSiNC [Alexa Fluor 488$\mathrm{mSiNC}]$ and determination of the fraction of $\mathrm{mSiNCs}$ that are actually fluorescent in Materials and methods; Fig. 5). The result showed that $38.4 \pm 2.6 \%$ of the mSiNCs were actually fluorescent (for six independently prepared specimens, examining 245 Alexa Fluor 488 spots in total), indicating much improvement from the previously reported value of $2.8 \%$, although the improvement mechanism is not clear.

\section{Extinction coefficient and quantum yield of mSiNCs}

FCS (Fig. 3 D) can determine the concentration of mSiNC particles that are actually fluorescent. Meanwhile, the result described in the previous subsection indicates that $38 \%$ of $\mathrm{mSiNC}$ particles fluoresce after they absorb light (making a plausible assumption that nonfluorescent particles have the same extinction coefficient as fluorescent ones). Using the absorption spectrum (Fig. 2 D, black solid line) and the concentration of $\mathrm{mSiNCs}$ (the concentration determined by FCS divided by 0.38 ), the extinction coefficients were obtained as $10.2( \pm 2.3) \times 10^{4}, 5.7$ $( \pm 1.1) \times 10^{4}$, and $5.3( \pm 1.4) \times 10^{4} \mathrm{M}^{-1} \mathrm{~cm}^{-1}$ at 350,400 , and $450 \mathrm{~nm}$, respectively $(n=12)$. This is the first determination of the SiNC extinction coefficients in an aqueous solution, and it shows that the mSiNC's extinction coefficient is comparable to those of protein fluorophores, although these values are substantially smaller than those for QDs (Table S1).

As another method to determine the extinction coefficient, we evaluated the molar concentration of $\mathrm{mSiNC}$ in aqueous solutions without relying on its optical properties (such as FCS and the fraction of actually fluorescent particles), we measured the actual weight of $\mathrm{mSiNC}$ after drying and estimated the molecular weight of the 3.2-nm mSiNC (36,083.6; see Determination of the amount of mercaptosilane bound to, and the extinction coefficient of, $\mathrm{mSiNC}$ in Materials and methods; in this process, the mean number of 3-MPTS molecules coating the mSiNC surface was determined as $51.2 \pm 19.4$ molecules/ $\mathrm{mSiNC}$ particle $[n=3])$. From the absorption spectrum obtained for these solutions, the extinction coefficients at 350,400, and $450 \mathrm{~nm}$ of the mSiNC solution were estimated to be $10.5( \pm 2.2) \times$ $10^{4}, 4.6( \pm 1.5) \times 10^{4}$, and $3.7( \pm 1.3) \times 10^{4} \mathrm{M}^{-1} \mathrm{~cm}^{-1}(n=3)$, 
A
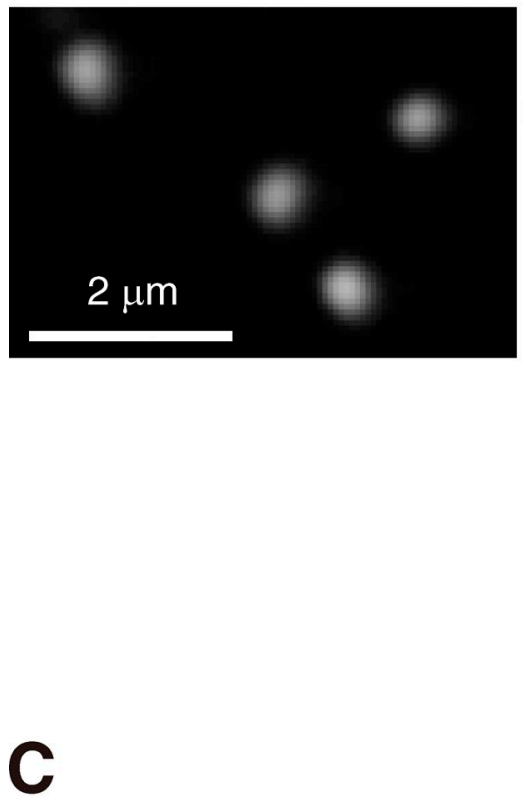

B

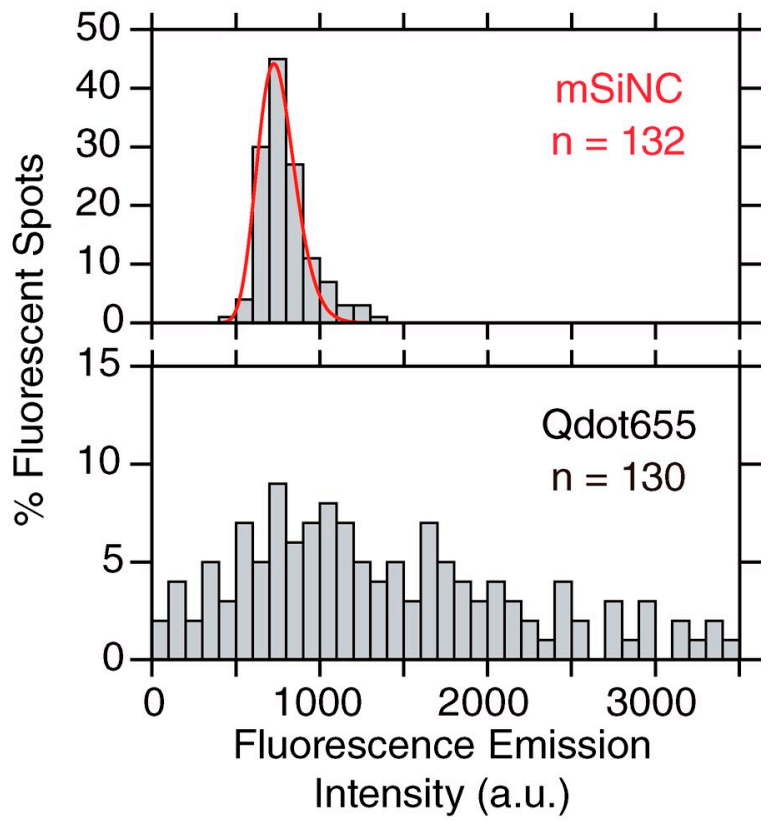

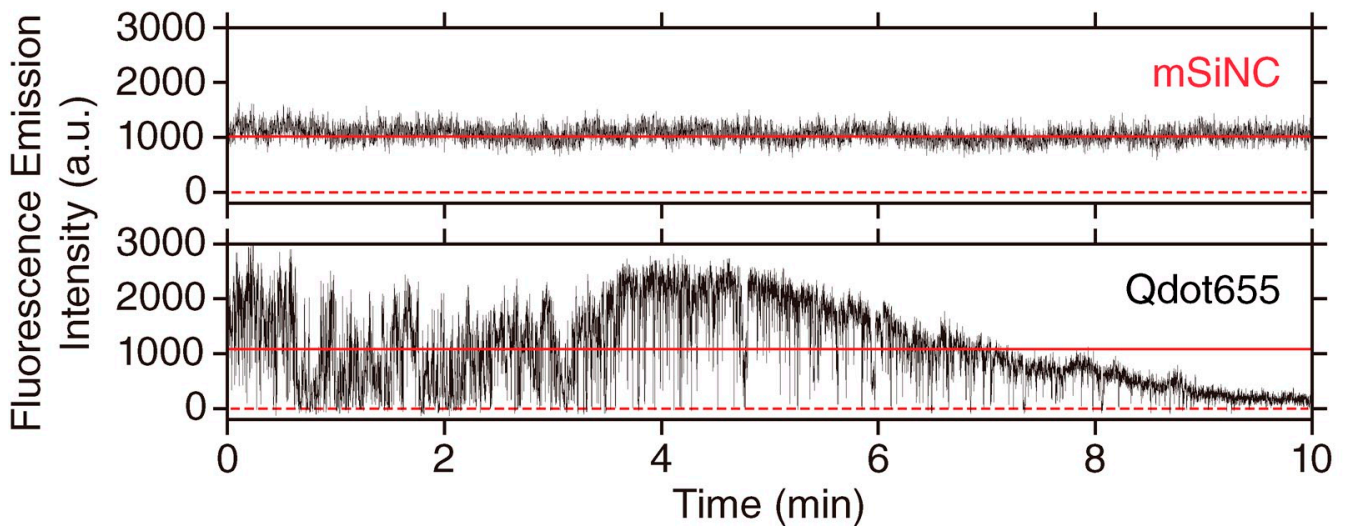

Figure 4. Single mSiNC tracking showed that, unlike QDs, $\mathrm{mSiNCs}$ neither blink nor photobleach over the total observation period of 300 min. (A) A typical TIRF microscope image of single HPLC-purified mSiNCs, immobilized on a polylysine-coated cover glass and excited by the 405-nm line of a solid-state laser. (B) Distribution of fluorescence intensities of $530 \times 530-\mathrm{nm}$ areas enclosing single $\mathrm{mSiNC}$ spots (top, mSiNC) and single Qdot655 (bottom, Qdot655). The fluorescence intensity is after background subtraction. The intensity distribution of mSiNC could be well fitted by a single log-normal function (red curve), and the possible dimer fraction was less than a few percentages, as determined by the two-component fitting, whereas the intensity distribution of Qdot655 was extremely broad. (C) Typical time-dependent changes of the fluorescence intensities of an mSiNC spot (top) and of a Qdot655 spot (bottom; typical among $30 \mathrm{mSiNC}$ and 30 Qdot655 particles, examined here. Other typical examples are shown in Fig. S3). Because of the background subtraction, the plot includes occasional negative signal intensities (0 is shown by red broken lines). The mean signal intensity is shown by solid red horizontal lines. Both fluorophores were excited by the same laser $\left(440 \mathrm{~nm}\right.$; $0.34 \mu \mathrm{W} / \mu \mathrm{m}^{2}$ at the sample plane). Images were recorded at video rate (33 ms/frame); see Video 1 and Fig. S3 A. The Qdot655 blinked frequently and photobleached gradually, whereas the mSiNCs never blinked during the video rate observations. Over $80 \%$ of the single Qdot655s observed in this study stopped emitting measurable fluorescence after the 10-min observation under these conditions. a.u., arbitrary unit.

respectively, in good agreement with the extinction coefficient determined from the FCS and the fraction of actually fluorescent particles.

The fluorescence quantum yield of the $\mathrm{mSiNCs}$ in $50 \mathrm{mM}$ phosphate buffer, $\mathrm{pH} 7.0$, was determined to be $0.080 \pm 0.0060$ $(n=6)$ (see Determining spectroscopic properties of mSiNC). Because this value was determined for the bulk mSiNC suspension (and thus is listed as bulk in Table S1), it includes mSiNCs that do not fluoresce even after absorbing light $(\sim 62 \%)$. The quantum yield of fluorescent $\mathrm{mSiNCs}$ was obtained by multiplication by $1 / 0.384$, giving a quantum yield of fluorescent $\mathrm{mSiNCs}$ of $0.21 \pm 0.016(n=6)$. When each individual $\mathrm{mSiNC}$ particle is to be observed, the quantum yield of 0.21 should be used, and therefore, it is listed as single particle in Table S1. As shown in Table S1, the quantum yield of mSiNC (0.21) is smaller than those for Qdot655 (>0.50) but is comparable with representative fluorescent organic Cy dye molecules. It is greater than the quantum yield of Cy3 (0.04) but smaller than that of Cy5 (0.27; measured in PBS; Mujumdar et al., 1993).

Based on the extinction coefficient and the quantum yield given in Table $\mathrm{S} 1$, Qdot655 is expected to be $\sim 200$-fold brighter than $\mathrm{mSiNCs}$ upon excitation at $440 \mathrm{~nm}$ (although the QDs' 

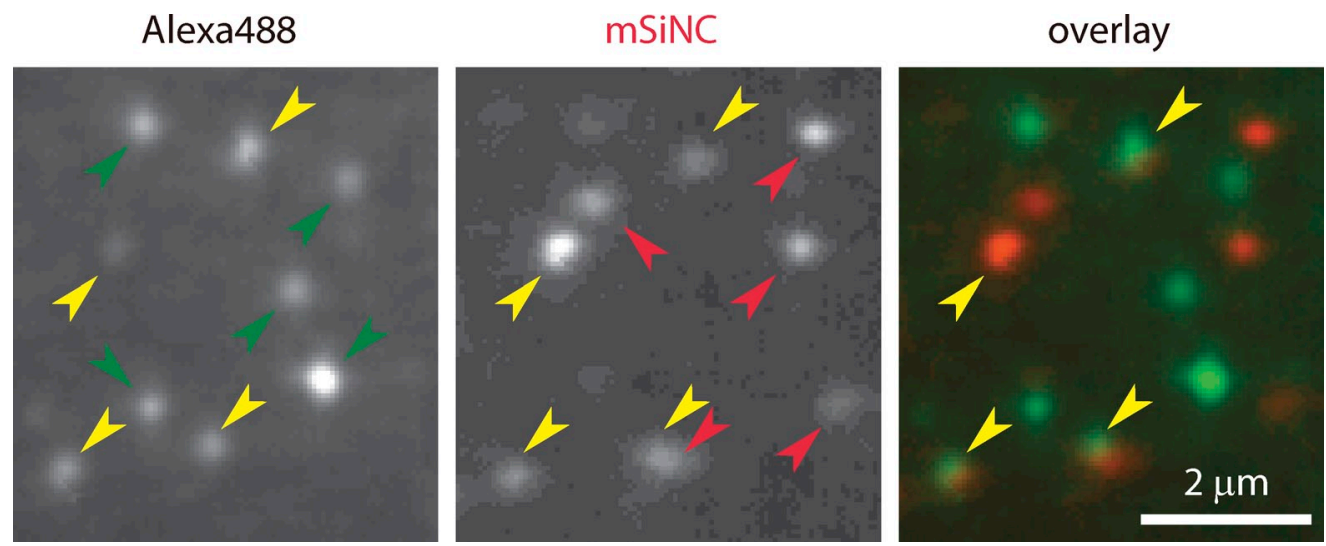

Figure 5. Simultaneous, dual-color fluorescence observations of single particles of Alexa Fluor 488-mSiNC, showing that $\sim 38 \%$ of $\mathrm{mSiNC}$ particles are fluorescent. Representative synchronously obtained, spatially corrected images of the Alexa Fluor488 (left) and the mSiNC (middle) and their overlaid images (right). Yellow arrowheads show colocalized spots (within $150 \mathrm{~nm}$ from each other). In the overlaid figure, apparently incomplete overlapping of green and red spots occurs (see the spot in the bottom left corner). However, these are within expected error range of $\sim 50 \mathrm{~nm}$ (Koyama-Honda et al., 2005). A spot with strong fluorescence intensity in the Alexa Fluor 488 channel probably represents an $\mathrm{mSiNC}$ that was conjugated with two or more molecules of Alexa Fluor 488 (this is expected to occur for $\sim 5 \%$ of the $\mathrm{mSiNC}$ particles). The stronger intensity spots in the mSiNC channel represent those at the high end of the intensity distribution shown in Fig. 4 B (top).

extinction coefficients from the supplier could not be verified in any refereed publications). However, as shown in Fig. $4 \mathrm{C}$ and Fig. S3, initially, the Qdot655 is brighter, but because it blinks frequently and its fluorescence intensity varies greatly with time, whereas the $\mathrm{mSiNC}$ fluorescence is stable over the same period, the fluorescence intensities of Qdot655 and mSiNC, averaged over the entire duration in which the Qdot655 emits a fluorescence signal (Fig. 4 C and Fig. S3, red, solid, horizontal lines), were similar to each other, under exactly the same illumination and detection conditions. Because single-molecule imaging has been widely conducted using, for example, EGFP (quantum yield $\varphi$ of $\sim 0.6$ and molar extinction coefficient $\varepsilon$ of $\sim 55,000 \mathrm{M}^{-1} \mathrm{~cm}^{-1}$; McRae et al., 2005) and $\mathrm{Cy} 3$ ( $\varphi$ of $\sim 0.04$ and $\varepsilon$ of $\sim 150,000 \mathrm{M}^{-1} \mathrm{~cm}^{-1}$; Table S1), it is not surprising that $\mathrm{mSiNC}$ ( $\varphi$ of $\sim 0.21$ and $\varepsilon$ of $\sim 55,000$ ) can be observed at the level of single particles. This suggests that the extinction coefficient of Qdot655 provided by the supplier must be cautiously reevaluated.

\section{Conjugating mSiNCs to proteins}

Using the protocol shown in Fig. 6 (A and B), mSiNC was conjugated to Tf, a serum protein that carries ferric ions into cells (see Preparation of mSiNC-labeled Tf [mSiNC-Tf] in Materials and methods). To examine the progress of the chemical conjugation, the reaction mixture of maleimide-conjugated $\mathrm{Tf}$ (maleimide-Tf) and $\mathrm{mSiNC}$ (2:1 mol ratio; 0 and $60 \mathrm{~min})$ was subjected to Superdex 200 gel filtration HPLC chromatography, and its elution pattern is shown in Fig. $6 \mathrm{C}$ (blue dextran represents the void volume). The $\mathrm{mSiNC}$ peak (Fig. $6 \mathrm{C}$, peak 3 ) decreases as the conjugation proceeds, whereas peak 2 grows and shifts toward larger molecular size. The behavior of the peak 2 (Fig. 6 C) suggests that the peak 2 after reaction for 60 min probably represents partially overlapped elution of $\mathrm{Tf}$ conjugated with mSiNC (mSiNC-Tf; larger) and maleimide-Tf (smaller). Peak 1 (Fig. 6 C) did not change. Because we suspected the presence of maleimide-Tf dimers in the reaction mixture, we generated artificial Tf dimers by a chemical cross-linker, bis(sulfosuccin imidyl)suberate, from $\mathrm{Tf}$ monomers ( $\mathrm{Tf}$ monomers and dimers were confirmed by SDS-PAGE) and examined the Superdex 200 chromatogram of the reaction mixture (Fig. $6 \mathrm{C}$, bottom). From this elution pattern, we concluded that peak 1 in Fig. $6 \mathrm{C}$ (top) represents maleimide-Tf dimers. Because the shape and the position of peak 1 did not change appreciably after the mSiNC conjugation reaction for $60 \mathrm{~min}$, it is concluded that $\mathrm{mSiNC}$ does not induce cross-linking of maleimide-Tf under these conjugation conditions.

Then, we tried to separate mSiNC-Tf and maleimide-Tf (overlapped peak 2 on the Superdex 200 chromatogram shown in Fig. 6 C, top) using Superdex 75. The Superdex 75 gel filtration HPLC chromatograms of the reaction mixture of maleimideconjugated Tf and $\mathrm{mSiNC}(2: 1 \mathrm{~mol}$ ratio; 0,30 , and $60 \mathrm{~min})$, shown in Fig. 6 D, reveal the progress of the chemical conjugation. The maleimide-Tf and $\mathrm{mSiNC}$ peaks (Fig. $6 \mathrm{D}$, peaks 2 and 3, respectively) decrease as the conjugation proceeds, whereas the new peak 1 (Fig. 6 D) at a larger molecular size appears and grows (the small peak eluted slightly after peak 1 at time 0 probably represents small amounts of contamination in the reaction mixture), suggesting that this new peak represents mSiNC-Tf.

Next, we examined whether peak 1 in the Superdex 75 chromatogram (Fig. 6 D) contained Tf molecules bound by multiple $\mathrm{mSiNCs}$. To investigate this possibility, we determined the number of mSiNCs attached to each Tf molecule. First, the mSiNC-Tf complexes recovered in peak 1 (Fig. 6 D) were adsorbed and immobilized on coverslips, and then, they were observed at the single-particle level using TIRF microscopy. The distribution of the signal intensity of each $\mathrm{mSiNC}$-Tf spot after 30- and 60-min reactions was the same as that for a single, unconjugated mSiNC (Fig. 6 E), and in all cases, the distributions could be fitted well with a single log-normal function (see the legend to Fig. 6 E) very similar to that shown in Fig. 4 B, showing that one mSiNC particle contributes to each mSiNC-Tf fluorescent spot.

These observations, together with the gel filtration HPLC results (Fig. 6, C and D), indicate that Tf precisely conjugated with 


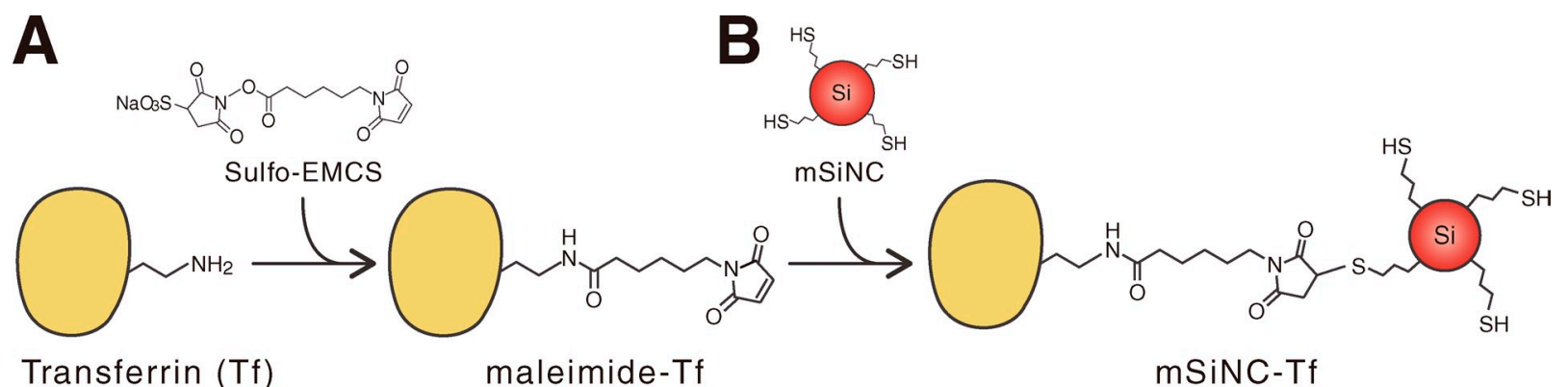

\section{Superdex-200}

$\mathbf{E}$

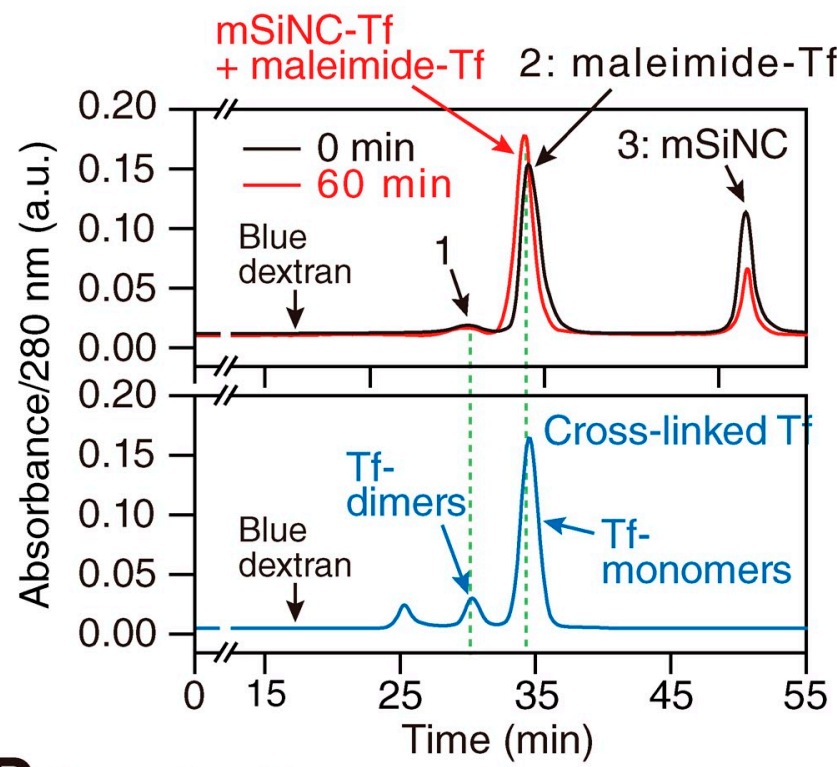

\section{Superdex-75}
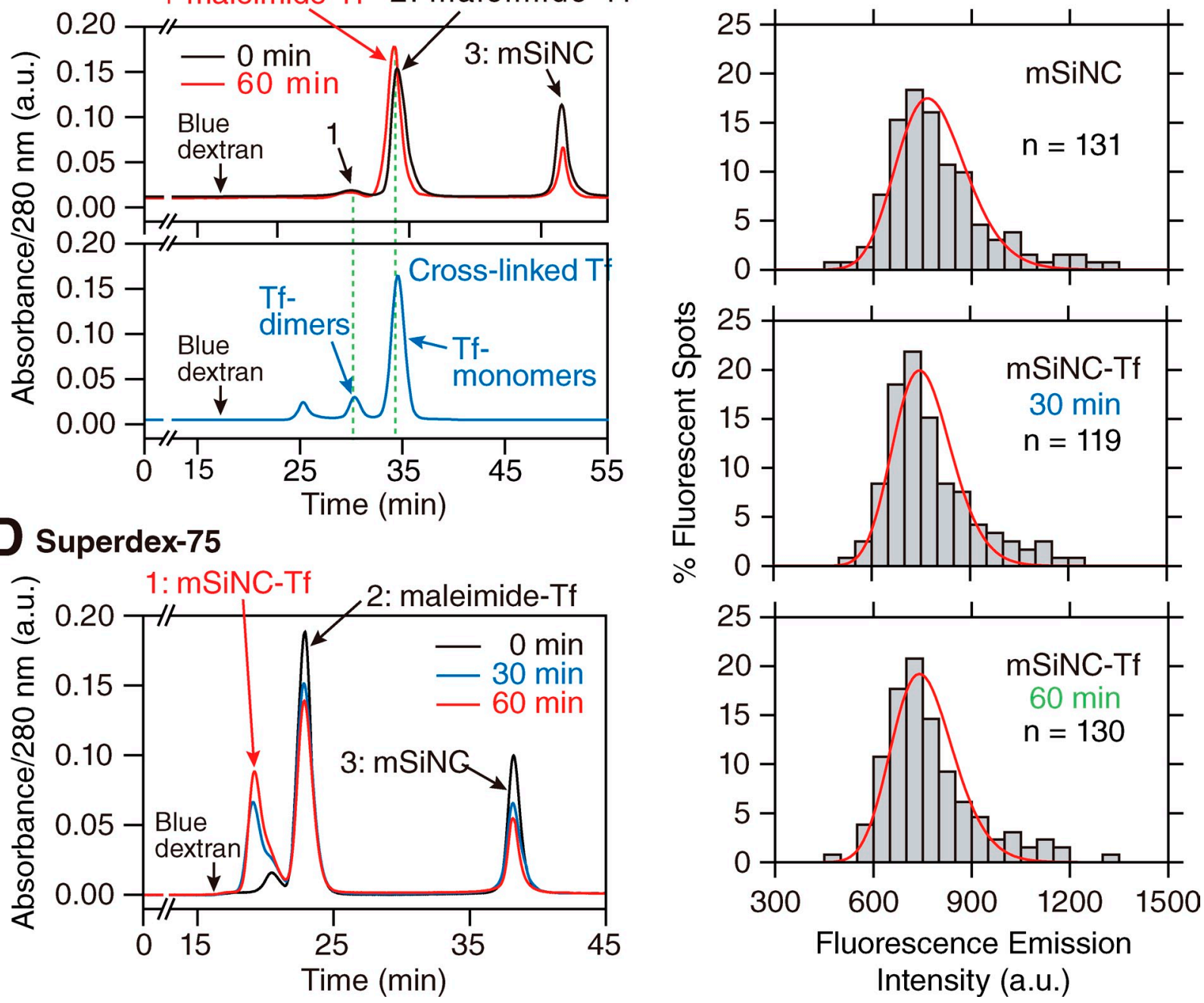

Figure 6. Conjugation of mSiNCs to Tf. (A and B) The scheme for conjugating mSiNC to Tf, using ( $N$ - $\varepsilon$-maleimidocaproyloxy) sulfosuccinimide ester (sulfoEMCS). (C, top) Superdex 200 gel filtration HPLC chromatograms for the samples obtained at 0 and 60 min after the mSiNC-Tf conjugation reaction was initiated. Peak 1, maleimide-Tf dimers (but not those cross-linked by mSiNC). Peak 2, overlapped eluent of mSiNC-Tf and maleimide-Tf. Peak 3 , unconjugated $\mathrm{mSiNC}$. The explanation for these assignments is given in the text. (bottom) Superdex 200 elution pattern of Tf monomers, dimers, and oligomers, generated by chemical cross-linking of Tf with bis(sulfosuccinimidyl)suberate. (D) Superdex 75 gel filtration HPLC chromatograms for the samples obtained at 0,30 , and 60 min after the mSiNC-Tf conjugation reaction was initiated. Peak 1, mSiNC-conjugated Tf (mSiNC-Tf). Peak 2, maleimide-Tf. Peak 3, unconjugated $\mathrm{mSiNCs}$. The explanation for these assignments is given in the text. Additionally, these chromatograms indicate that about half of the mSiNCs added for this conjugation reaction became conjugated to maleimide-Tf in $60 \mathrm{~min}$. Note that all of these peaks occur after the void volume determined by Blue Dextran 2000. Data in early time periods were deleted in C and D because no elvent peaks were detectable during these early periods. (E) Distributions of fluorescence intensities of individual mSiNC or mSiNC-Tf spots (measured in $530 \times 530-\mathrm{nm}$ areas) after the conjugation reactions for 0,30 , and $60 \mathrm{~min}$. Excitation was by the 405-nm line of a solid-state laser. A single log-normal function (red curves) gave a good fit, and the two-component fitting did not significantly improve the fit, but nevertheless, the latter gave an estimate of the possible dimer fraction of less than a few percentages. Typical results among $7(C$, top), $3(C$, bottom), $11(D)$, and $6(E)$ entirely independent experiments are shown. a.u., arbitrary unit. 

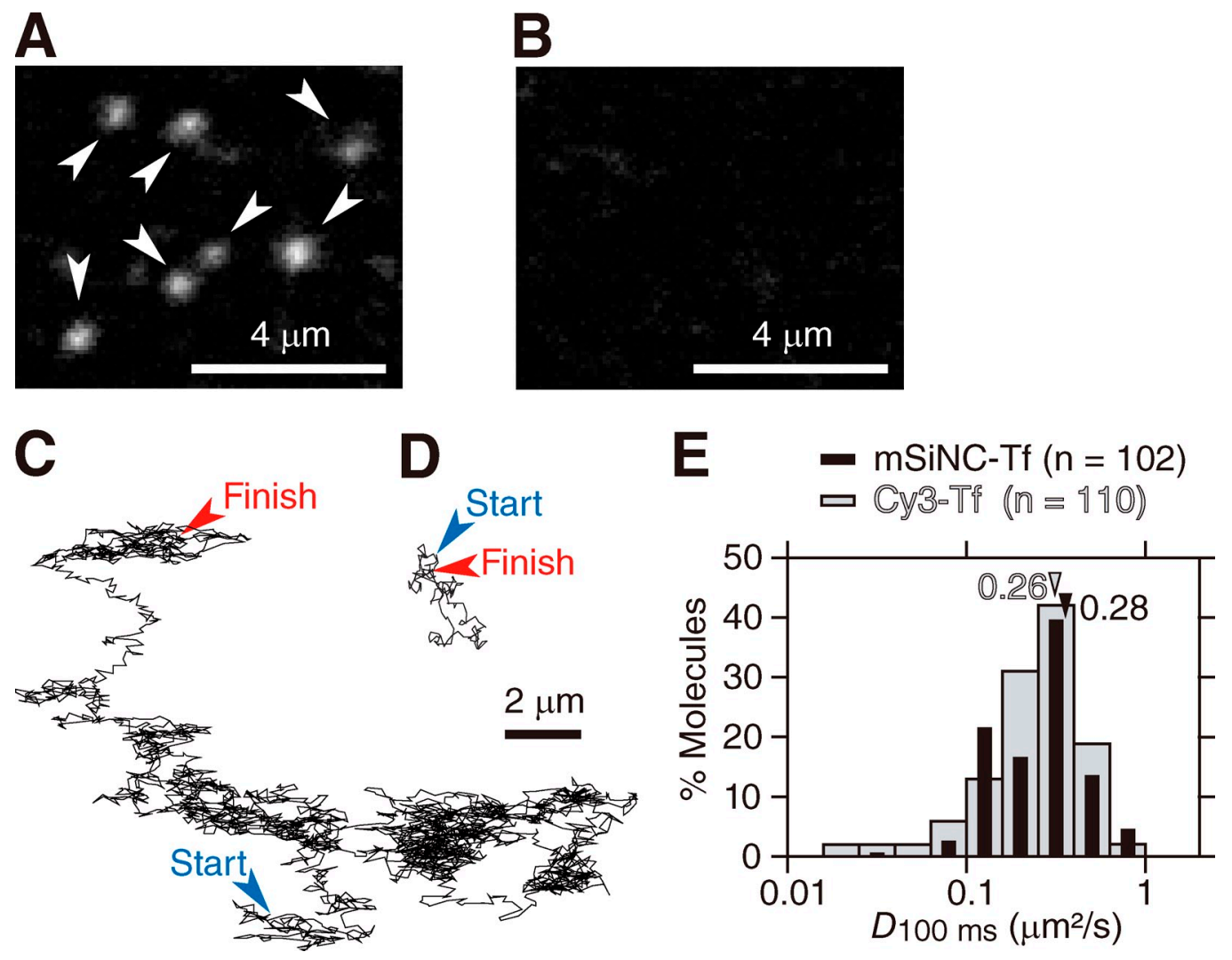

Figure 7. Tracking of each individual TfR molecule on the plasma membrane of live NRK cells, using mSiNC-Tf. (A and B) Single-particle images of $m$ SiNCTf (human Tf used throughout this study), showing the binding and nonbinding of mSiNC-Tf to the bottom surfaces of a single NRK cell (arrowheads; see Video 2; A) and a single CEF cell (B), respectively. Human Tf has been known to bind to rat TfR, but not chick TfR, and thus, B serves as a negative control for A. (C) A typical trajectory ( 122 s and 3,651 frames) of a TfR molecule tagged by an mSiNC-Tf, observed in the bottom plasma membrane of an NRK cell at video rate (33 ms/frame). (D) A typical trajectory $(5 \mathrm{~s}$ and 150 frames) of a TfR molecule conjugated with Cy3-Tf, obtained as in D. (E) The distribution of the diffusion coefficient of TfR in the time scale of $100 \mathrm{~ms}$, determined with mSiNC-Tf, is similar to that determined with Cy3-Tf. The numbers in the box indicate median values (see arrowheads).

a single $\mathrm{mSiNC}$ was prepared and that $\mathrm{mSiNC}-\mathrm{Tf}$ is monodisperse. Labeling of a protein with a nanocrystal at the $1: 1$ ratio with this precision now makes high-definition single-molecule tracking possible.

\section{Single-molecule tracking of Tf receptor [TfR] on the plasma membrane with mSiNC-Tf}

Each individual mSiNC-Tf bound to the bottom surface of the normal rat kidney (NRK) cell was observed with a TIRF microscope (both Cy3-labeled Tf [Cy3-Tf] and mSiNC-Tf readily enter the gap between the bottom membrane and the coverslip, practically reaching the equilibrium binding within a minute; Kasai et al., 2011; Fig. 7 A) at $37^{\circ} \mathrm{C}$. The specific binding of mSiNC-Tf to TfR in the plasma membrane was confirmed by the following four experiments. (1) The mSiNCs that had not been conjugated to Tf did not bind to the cultured NRK cells $(<1 / 100)$. (2) The inclusion of $100 \mathrm{nM}$ free $\mathrm{Tf}$ in the incubation medium containing mSiNC-Tf strongly ( $90 \%$ ) inhibited $\mathrm{mSiNC}$-Tf binding to the cell surface, suggesting that $\sim 10 \%$ of mSiNC-Tf bound to the cell surface is nonspecifically adsorbed, consistent with the data of colloidal gold-conjugated molecules ( $~ 95 \%$; Kusumi et al., 1993). (3) The mSiNC-Tf developed here, using human Tf, did not bind to the surface of chick embryonic fibroblasts
(CEFs; Fig. 7 B). Because it has been shown that human Tf does not bind to chick TfR (Jing et al., 1990), this lack of binding further confirmed the specificity of mSiNC-Tf binding to TfR molecules on NRK cells (human Tf binds to rat TfR; Jing et al., 1990). (4) When mSiNC-Tf was applied to the culture of CEFs transfected with the GFP-human TfR (hTfR) gene, mSiNC-Tf bound only to the cells that expressed GFP-hTfR protein (the signal looks weak because the general staining of the cells was observed by epifluorescence microscopy; Fig. S5 A).

The single TfR-mSiNC-Tf complex could be tracked at video rate, without blinking or photobleaching, for $\leq 3,600$ frames or $120 \mathrm{~s}$ (Fig. 7 C). Photobleaching prevented Cy3-Tf from being tracked for longer than 150 frames ( $5 \mathrm{~s}$; Fig. 7 D), whereas blinking prevented the tracking of GFP-tagged molecules longer than 20 frames $(\sim 0.67 \mathrm{~s}$; both in the case in which no reconnection protocol is used; see the next paragraph), indicating improvements by one to two orders of magnitude.

In the majority of single fluorescent molecule-tracking experiments reported in literature, even if the fluorescence signal from the probe disappears in several or many consecutive video frames (gap), the two trajectories separated by a gap are often "reconnected" into a single trajectory, assuming that the disappearance of the signal is caused by blinking (Table S2). This 
is how trajectories longer than the blinking time were obtained with the use of GFP-type molecules, QDs, and some organic dyes, such as Alexa Fluor 488 and Alexa Fluor 594.

In the present experiment, we aborted the tracking if we missed the observed spot even for a single video frame, i.e., no reconnection was performed. Even under such rigorous conditions, we were able to track single TfR molecules, tagged with $\mathrm{mSiNC}$-Tf, for up to $\sim 3,600$ frames ( $120 \mathrm{~s}$ ). This is about twice as long as the longest observation frames ever made using extensive reconnection protocols (Table S2). Note that, because the overall observation durations can be prolonged by time lapsing, the number of observed frames, and not the observed time period, is the critical parameter.

The failure to detect a fluorescent spot of an mSiNC particle in a single (or more) frames appears to occur for the following reasons: (a) temporary blurring of the fluorescent spots, as a result of z-directional motion, (b) release of the mSiNC-Tf from the receptor, (c) normal internalization processes of TfR molecules, and (d) long-term instability of the focus at $37^{\circ} \mathrm{C}$, raised from the room temperature. With the use of mSiNCs, photobleaching and blinking are no longer the limiting factors for the durations of single-molecule tracking in living cells. Phototoxicity of the 440-nm laser illumination to the cell was not apparent, at least for $5 \mathrm{~min}$ (see Cell preparation for fluorescence microscopy in Materials and methods).

A statistical analysis (Kusumi et al., 1993) indicated that virtually all of the TfR molecules probed with mSiNC-Tfs at video rate undergo (apparent) simple Brownian diffusion (Fujiwara et al., 2002). The distribution of the effective diffusion coefficients in the time scale of $100 \mathrm{~ms}$ (Kusumi et al., 1993) is shown in Fig. 7 E. The median diffusion coefficient was $0.28 \mu \mathrm{m}^{2} / \mathrm{s}$ ( $n=102)$, which is comparable with that found with single Tf molecules tagged with Cy3 (median, $0.26 \mu \mathrm{m}^{2} / \mathrm{s}, n=110$; insignificant statistical difference). These results suggest that $\mathrm{mSiNC}$ Tfs are bound 1:1 to the TfR molecules without cross-linking them, and mSiNC-Tfs themselves are not clustered (otherwise, mSiNCTf would have shown much slower diffusion by oligomerizationinduced trapping; Iino et al., 2001), thus allowing the accurate observation of single-molecule diffusion of TfR.

Another representative type of the trajectories is shown in Fig. S5 B. After undergoing simple Brownian diffusion for $12.4 \mathrm{~s}$, this TfR molecule underwent temporary immobilization for $1.7 \mathrm{~s}$, as detected by a computer program (Fig. S5 C; Simson et al., 1995), and then disappeared from the TIRF image (Fig. S5 B), suggesting the entrapment of TfR-mSiNC-Tf complex in a clathrin-coated pit, followed by internalization (Video 3). Previously, direct observation of a single molecule's diffusion in the general plasma membrane area followed by its entrapment and internalization had never been achieved as a result of photobleaching in the case of organic dyes and GFP and perhaps because of slowed internalization of the receptor linked to a large particle, such as QDs and colloidal gold particles. Such direct observations of single receptor molecules undergoing diffusion in the plasma membrane and the subsequent internalization will further advance the studies about the mechanism of liganded receptor internalization and signaling in the intracellular membrane systems.
Spatial variations of the TfR diffusion coefficient on the cell surface

Spatial variations of the physical properties of the plasma membrane have long been suspected. In the context of signal transduction function of the plasma membrane, spatial variations might be useful for creating space-dependent functional specializations, leading to, for example, polarized responses of the cells and/or enhanced interactions of molecules in specific locations in the plasma membrane (Chung et al., 2010). One of the useful parameters for describing the spatial variations is the diffusion coefficient of molecules, which might reflect the spatial variations in the interaction between the actin-based membrane skeleton meshwork and the plasma membrane, the densities of raft domains in the plasma membrane, and the densities of the binding sites for membrane molecules under investigation on the cortical actin filaments. However, spatial variations of the diffusion coefficients have not been systematically investigated. Such spatial variations can now be investigated with high reliability using a long trajectory of each individual molecule, which continuously explores various domains in the plasma membrane, reporting spatial variations of its local diffusion coefficient.

To obtain the spatial variations of the local diffusion coefficient, we used the display shown in Fig. 8 B (top; for the trajectory shown in Fig. $8 \mathrm{~A}$ ), in which cumulative square displacement (SD; CSD; the sum of the SD taken every $33 \mathrm{~ms}$ ) is plotted against time, with a relationship of CSD $=4 D t$. Namely, the local slope/4 gives the local diffusion coefficient. Therefore, the changes in diffusivity could be identified in the plot, as the time intervals with different slopes (Chung et al., 2010). In this analysis, we imposed $5 \mathrm{~s}$ as the minimum duration for a given diffusivity state, within which any possible change in diffusion dynamics is ignored because a small number of plots leads to increasing uncertainty in the observations (see Detection of transient confinement zones and time [area]-dependent changes of diffusion coefficient in Materials and methods). In short, the long-term (for this study $>5 \mathrm{~s}$ ) local diffusion coefficient for the specific time period could be directly obtained from this plot (Fig. 8 B, bottom). From these displays, we understand that, even during the overall simple Brownian diffusion period, single TfR molecules can exhibit periods ( $>5 \mathrm{~s}$ ) when they diffused at much smaller rates (Fig. $8 \mathrm{~B}$, blue and red regions) or greater rates (Fig. $8 \mathrm{~B}$, green region) than the average diffusion rate in a domain of $1-2 \mu \mathrm{m}$ in diameter. Our attempt to find any correlation of slow- or fast-diffusion domains with the features that appeared in the enhanced bright-field images was not successful.

To further quantitate this observation, the distribution of long-term ( $>5 \mathrm{~s}$, but still local) diffusion coefficients of TfR was obtained (Fig. $8 \mathrm{C}$, open bars). This distribution is compared with that for the diffusion coefficient determined on a $100-\mathrm{ms}$ scale (Fig. $8 \mathrm{C}$, solid black bars). The average diffusion coefficient in the long-time regimen (median $\left.=0.19 \mu \mathrm{m}^{2} / \mathrm{s} ; n=45\right)$ is $\sim 30 \%$ smaller than that in the short-time regimen $(\sim 100 \mathrm{~ms}$; median $=0.28 \mu \mathrm{m}^{2} / \mathrm{s} ; n=102$ ), whereas the spread in the longtime regimen (a factor of several hundred, $\mathrm{SD}=0.27 \mu \mathrm{m}^{2} / \mathrm{s}$; particularly, note the long tails toward the smaller values in the distribution) is much greater than that in the short-time regimen (a factor of $\sim 20 ; \mathrm{SD}=0.16 \mu \mathrm{m}^{2} / \mathrm{s}$ ), as shown in Fig. $8 \mathrm{C}$. These 


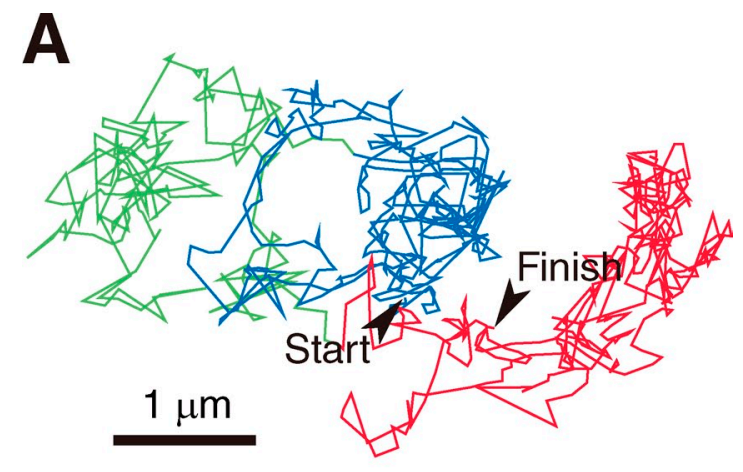

B
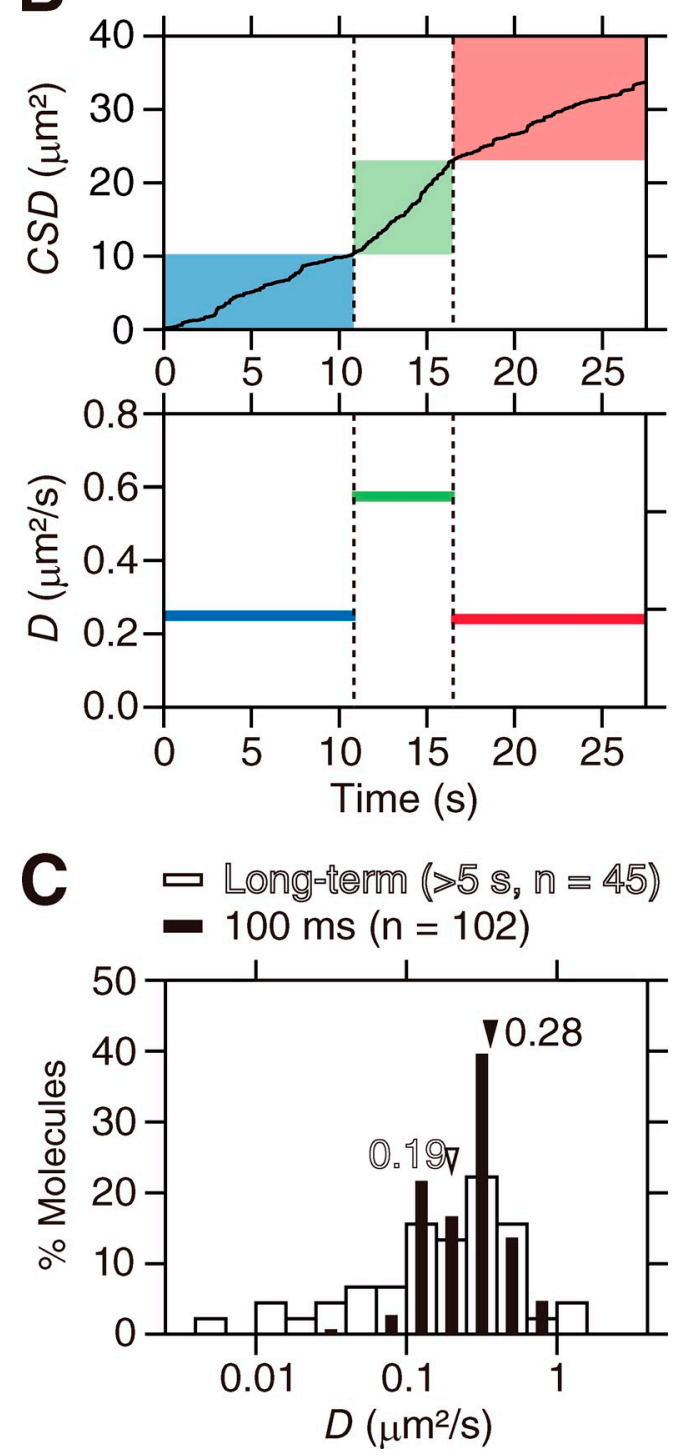

Figure 8. Large variations of the long-term ( $>5 \mathrm{~s}$ ) diffusion coefficient experienced by single molecules of TfR on the cell surface. (A) A typical trajectory of a TfR molecule tagged by $\mathrm{mSiNC}$-Tf, recorded at video rate (33 ms/frame). Different colors indicate the portions of the trajectory with different diffusivity, as determined by the method described in B. (B) Quantitative analysis results of the trajectory shown in A. (top) The CSD plotted as a function of time. The plot is divided into subintervals, in which a single slope can describe the plot, by eye, and if the portion is longer than $5 \mathrm{~s}$, its slope was obtained, as plotted at the bottom. (C) Distribution of the longterm (>5 s) diffusion coefficients, indicating the presence of long-range spatial variations in the membrane structure, probably reflecting local results can be explained in the following way. TfR molecules crisscross at faster rates (and thus the diffusion coefficient in the 100 -ms regimen is greater) without undergoing long-range diffusion (staying in a region of $\sim 200 \mathrm{~nm}$ or less), probably confined within one or two compartments made by the partitioning by the actin-based membrane skeleton (fences) and its associated transmembrane proteins (pickets) or linked to the actinbased membrane skeleton that is undergoing rapid thermal fluctuation (Fujiwara et al., 2002; Kusumi et al., 2005), which could not be resolved at video rate $(33 \mathrm{~ms})$. Meanwhile, in the long-time regimen ( $>5 \mathrm{~s}$ ) in which the macroscopic long-range diffusion matters, the diffusion of TfR appears slower because it experiences various diffusion barriers and temporary bindings during this period (but again each event could not be resolved at this time resolution and thus appears as decreases in the mean long-term diffusion coefficient), and the long-term diffusion coefficients vary greatly because, during the periods of $>5 \mathrm{~s}$, TfR molecules would experience many barriers, obstacles, and traps that vary greatly in numbers and properties depending on the location (micrometer level) of the TfR molecule in the plasma membrane. To the best on our knowledge, this is the first time that the diffusion coefficient in the time scale of $5 \mathrm{~s}$ or longer was measured for each individual molecule and was shown to spatially vary greatly (for exactly the same molecule), as much as a factor of several hundred.

\section{Discussion}

The problems of photobleaching, blinking, and cross-linking that fluorescent probes often exhibit are particularly severe in the studies using living cells, in which reducing-oxidizing chemicals cannot be used because of their toxic side effects, molecular oxygen cannot be removed, and the effects of cross-linking are severe. The mSiNCs developed in this study were subjected to most stringent tests of single-molecule imaging as well as many other characterizations and found to simultaneously solve many problems generally associated with fluorescent particles, dyes, and proteins. mSiNCs neither bleach nor blink at least for the total observation period of $300 \mathrm{~min}$ (540,000 frames) and are small (uniformly $\sim 4 \mathrm{~nm}$ including the coat; although this size is greater than those of organic dye molecules) and monodisperse; they can be treated as water-soluble, monodispersed particles like highly soluble proteins, and a 1:1 conjugation of an $\mathrm{mSiNC}$ particle to a protein molecule was realized. When labeled with single $\mathrm{mSiNCs}$, single receptors in the plasma membrane of living cells could be tracked for $\sim 3,600$ image frames, without missing it even for a single frame. This limitation was not caused by either blinking or photobleaching of the mSiNC. Phototoxicity of the 440-nm laser illumination to living cells was not detectable at least for $5 \mathrm{~min}$ in single $\mathrm{mSiNC}$ particle

variations in the actin-based membrane skeleton (open bars; obtained for 30 TfR molecules; 45 separate subintervals exhibiting different diffusion coefficients in time). This should be compared with the histogram of 100-msscale diffusion coefficients (black bars, $n=102$ ). The numbers in the box indicate median values (see arrowheads). 
tracking. Because mSiNCs pass the rigorous tests for singlemolecule tracking, they will be useful for general fluorescence imaging of live and fixed cells.

Both QDs and mSiNCs have their advantages and disadvantages. We envisage that $\mathrm{mSiNCs}$ will revolutionize singlemolecule tracking in living cells. Meanwhile, for a few minutes tracking of membrane-bound receptors that diffuse slowly or are immobilized, blinking might not strongly affect the tracking, and therefore, QDs might be preferable as a result of more color selections (particularly for multicolor tracking) and shortterm brightness. The availability of two different types of fluorescent biocompatible nanoparticles would be good for the fluorescence imaging community.

Using mSiNC-Tf, we were able to specifically label TfR and tracked the movement of the TfR-mSiNC-Tf complex on the NRK cell surface. Using mSiNC-Tf (1:1 conjugation) allowed one for the first time ever to directly observe, at the level of single receptor molecules, the initial entrapment of TfR within a clathrin-coated pit and the subsequent internalization process of each individual molecule. This was made possible by $\mathrm{mSiNC}$ because it does not photobleach or blink and is sufficiently small to allow internalization at rates comparable to physiological ligands. Therefore, mSiNC-conjugated ligands will make useful fluorescent markers to investigate their receptors' internalization mechanisms by way of clathrin-coated pits and possibly of other domains, such as caveolae and GEECs (Chadda et al., 2007).

Furthermore, spatial variations of the diffusion coefficients of molecules in the plasma membrane, which have been sought after for a long time, have been discovered by using TfR labeled with $\mathrm{mSiNC}$-Tf. This result is very reliable because spatial variations were found by single molecules that explored large areas on the cell surface, which the development of mSiNC-Tf made possible. Single TfR molecules (TfR-mSiNC-Tf complexes) exhibited various diffusion coefficients in membrane domains

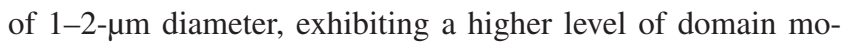
saicism in the plasma membrane, in addition to raft domains (3-100 nm) and membrane compartmentalization (30-300 nm) provided by the membrane skeleton (fence) and its associated transmembrane proteins (pickets; Fujiwara et al., 2002; Kusumi et al., 2005). The micrometer-level spatial variations might be caused by those of the actin-based membrane skeleton meshwork (spatial variations in mesh sizes and actin filament dynamics) as well as to spatial variations of the densities of raft domains and transient TfR binding sites. Spatial variations of the diffusion coefficient in the scale of $1 \mu \mathrm{m}$ or longer might be useful for inducing polarized signal transduction and local cytoskeletal reorganization upon stimulation. These will be clarified in the near future by tracking long-term movements of various receptors tagged by $\mathrm{mSiNCs}$.

One obvious application of mSiNCs will be their use as fiducial markers in single-molecule localization microscopy. $\mathrm{mSiNC}$ can be readily excited at the 405-nm laser line used for photoactivation photoconversion, and its fluorescence is detectable in broad regions between 500 and $700 \mathrm{~nm}$, where fluorescence signal detection for localization microscopy is generally conducted. Overall, in this research, we took advantage of the fluorescent SiNCs developed in physical and chemical laboratories and developed truly useful SiNC-based fluorescent probes for cell-biological and biomedical research, particularly for singlemolecule imaging and tracking.

\section{Materials and methods}

\section{Preparation of $\mathrm{mSiNC}$ and $\mathrm{mSiNC}$-labeled Tf}

Preparation of SiNC on the wafer. All the procedures for preparing mSiNC were conducted in the fume hood, and the relative humidity of the room was kept at $<65 \%$. All reagents, including $\mathrm{HF}, \mathrm{HNO}_{3}$, acetic acid, and methanol, were of the reagent grade or better and were used without further purification and/or dehydration. A single-crystal silicon wafer ((100) orientation, 1-10 $\Omega-\mathrm{cm}$ resistivity, and p-type boron doped; Mitsubishi Materials) was broken into smaller pieces (approximately $3.5 \times 4.0 \mathrm{~cm}$; Fig. $1 \mathrm{~A}$, left). Etching was performed by immersing one side of the piece $\sim 2 \mathrm{~mm}$ deep in $\mathrm{HF} / \mathrm{HNO}_{3}(1: 1, \mathrm{vol} / \mathrm{vol})$ along the surface of the wafer (Fig. $1 \mathrm{~A}$, second from the left) for a few seconds, to generate starting points for etching (preetching). The entire piece was then immersed in a $\mathrm{HF} / \mathrm{HNO}_{3} / \mathrm{H}_{2} \mathrm{O}$ solution $(2: 7: 8, \mathrm{vol} / \mathrm{vol} / \mathrm{vol})$ for $5 \mathrm{~min}$ (Fig. $1 \mathrm{~A}$, third image). This etched wafer (wSiNC) was washed thoroughly in water purified by an ion-exchange resin and a reverse osmosis system (Milli-Q; EMD Millipore) and then was dried under vacuum $(\sim 0.1 \mathrm{~mm} \mathrm{Hg})$.

Surface silanization of the SiNC on the wafer (wSiNC) with mercaptosilane. Silanization was performed immediately after the etched wafers were dried (Fig. 1 B). Each wafer was submerged for $1.5 \mathrm{~h}$ in an acidic methanol solution ( $0.5 \mathrm{ml}$ of 12-M acetic acid mixed with $500 \mathrm{ml}$ methanol) containing 1\% (vol/vol) 3-mercaptopropyltrimethoxysilane (Chisso). In this solution, 3-mercaptopropyltrimethoxysilane is hydrolyzed to produce 3-MPTS. After silanization, the wafers were washed thoroughly in methanol and then were dried under vacuum $(\sim 0.1 \mathrm{~mm} \mathrm{Hg})$.

Recovery and purification of mSiNC. Nanocrystals were liberated from the polished side of the wafers by mechanical scraping of the silanizedetched surface using a razor blade and were suspended in water ( $\mathrm{mSiNC}$; Fig. $1 \mathrm{C}$ ). The suspension was sonicated for $2 \mathrm{~h}$ and was centrifuged at $12,000 \mathrm{~g}$ for $30 \mathrm{~min}$. The $\mathrm{mSiNC}$ in the supernatant was further purified by an HPLC system (Hitachi) with a Superdex 75 column 10/300 GL (GE Healthcare) equilibrated in $50 \mathrm{mM}$ phosphate buffer, $\mathrm{pH} 7.0$, at a flow rate of $0.4 \mathrm{ml} / \mathrm{min}$. Elution profiles were monitored by recording the absorption at $280 \mathrm{~nm}$.

In a single preparation of $\mathrm{mSiNC}$, we typically obtained $\sim 2 \mathrm{nmol}$ (typically $\sim 1 \mu \mathrm{M} \times 2 \mathrm{ml}$ ) of $\mathrm{mSiNC}$ from a 6-inch wafer. This is comparable to a single commercial vial of Qdot655, which contains $\sim 2 \mathrm{nmol}$ (typically $\sim 8 \mu \mathrm{M} \times 0.25 \mathrm{ml}$ ) of $Q \mathrm{dot} 655$. A project for scaling up the quantity of $\mathrm{mSiNC}$ in each preparation is in progress in our laboratories, and at the level of a basic science laboratory, a 30-fold increase appears to be achievable. Therefore, in the near future, we should be able to provide interested researchers with $\mathrm{mSiNC}$ for testing its applicability to their own projects. However, for the preparations at the industrial level, greater efforts would be required.

Preparation of mSiNC-labeled Tf (mSiNC-Tf). Tf was labeled with $\mathrm{mSiNC}$, following the procedure illustrated in Fig. 6 ( $\mathrm{A}$ and $\mathrm{B}$ ). For the production of maleimide-Tf (Fig. 6 A), $20 \mu \mathrm{N}$-( $\varepsilon$-maleimidocaproyloxy)sulfosuccinimide ester (sulfo-EMCS; $10 \mathrm{mM}$; Thermo Fisher Scientific) in PBS, $\mathrm{pH} 7.2$, was added to $200 \mu \mathrm{lff}$ (holo-Tf, human; $0.1 \mathrm{mM}$; Sigma-Aldrich) in PBS, $\mathrm{pH} \mathrm{7.2,} \mathrm{and} \mathrm{the} \mathrm{mixture} \mathrm{was} \mathrm{incubated} \mathrm{at} \mathrm{room} \mathrm{temperature} \mathrm{for}$ $60 \mathrm{~min}$. Sulfo-EMCS-conjugated Tf (maleimide-Tf) was separated by chromatography on a Sephadex G-25 (GE Healthcare) column equilibrated with PBS. The maleimide/Tf molar ratio was adjusted to be low, i.e., $0.41 \pm$ 0.093 (mean $\pm S D, n=10$; the maleimide concentration in the maleimideTf solution was determined in the following way. $40 \mu \mathrm{l}$ mercaptoethylamine [0.125 mM in $50 \mathrm{mM}$ EDTA, pH 6.0] was first mixed with $100 \mu$ of purified maleimide-Tf $[0.01 \mathrm{mM}]$, and the mixture was incubated at $30^{\circ} \mathrm{C}$ for $30 \mathrm{~min}$, and then, the reduction of the thiol group in the mixture was determined by a reaction with $10 \mu \mathrm{l} 44^{\prime}$-dithiodipyridine $\left[5 \mathrm{mM}\right.$; at $30^{\circ} \mathrm{C}$ for 15 min; Grassetti and Murray, 1967] using the mercaptoethylamine reacted [room temperature for $60 \mathrm{~min}$ ] with 1-64 $\mu \mathrm{M}$ sulfo-EMCS preincubated in water for $3 \mathrm{~h}$ to hydrolyze the succinimide group as standard solutions. The reaction was monitored by the optical density at $324 \mathrm{~nm}$ $\left[\varepsilon=19,800 \mathrm{M}^{-1} \mathrm{~cm}^{-1}\right]$ ). Assuming the Poisson distribution with a mean value of 0.41 maleimide group/Tf, $\sim 6 \%$ of Tf should be conjugated with two or more maleimide groups.

To $40 \mu$ of 10- $\mu \mathrm{M}$ maleimide-Tf in PBS, $\mathrm{pH} 7.2$, we added $200 \mu \mathrm{l}$ of $1-\mu \mathrm{M} \mathrm{mSiNC}$ and incubated the mixture at room temperature for 30 and 
60 min. Tf labeled with mSiNC was separated by gel filtration HPLC (Superdex 75 and Superdex 200 columns [GE Healthcare] equilibrated with $50 \mathrm{mM}$ phosphate buffer, $\mathrm{pH} 7.0$ ) from the nonlabeled Tf and the free mSiNC (Fig. 6, C-E).

In this reaction, the maleimide-Tf/mSiNC ratio in the reaction mixture was $\sim 0.82$ (because maleimide/Tf ratio is 0.41 and $\mathrm{Tf} / \mathrm{mSiNC}$ ratio is 2 ). As seen in the peaks 2 and 3 in Fig. 6 D, 20\% of maleimide-Tf + nonlabeled $\mathrm{Tf}$, i.e., $\sim 50 \%$ of maleimide-Tf and $\sim 40 \%$ of $\mathrm{mSiNC}$, were consumed to form $\mathrm{mSiNC}$-Tf within $60 \mathrm{~min}$, providing a mean $\mathrm{mSiNC} / \mathrm{Tf}$ ratio of $\sim 0.4$. Assuming the Poisson distribution with a mean value of $0.4 \mathrm{Tf}$ molecules/ $\mathrm{mSiNC}$ particles, $\sim 6 \%$ of $\mathrm{mSiNC}$ should be conjugated with two or more molecules of Tf. That is, despite the presence of many sulfhydryl groups on the mSiNC, we can achieve almost 1:1 conjugation of Tf to mSiNC, particularly after the HPLC purification (Fig. $6 \mathrm{D}$, peak 1). mSiNCs conjugated with multiple If molecules were not detectable in the chromatogram shown in Fig. $6 \mathrm{D}$ probably because their peaks were broad, overlapped, and small.

\section{Characterization of $\mathrm{mSiNC}$ and $\mathrm{mSiNC}$-Tf}

Determining the surface coating of wmSiNC and mSiNC. A suspension of $\sim 4$-nmdiameter $\mathrm{mSiNC}$, purified by Superdex 75 gel filtration HPLC, was dialyzed against $50 \mathrm{mM} \mathrm{NH}_{4} \mathrm{Cl}$ using 3,500 molecular weight cut-off dialysis cassettes (Slide-A-Lyzer; Thermo Fisher Scientific). The surface coatings of the wmSiNC (mercaptosilane-coated SiNC on the wafer) and the mSiNC (dispersed nanocrystals in the dialysis solution were placed on the $\mathrm{KBr}$ plate and dried under ambient conditions) were analyzed by a spectrometer (1,024 acquisitions for a single spectrum; FTS-3000 FTIR; Varian). Background spectra were obtained using uncoated SiNC on wafers (wSiNC) or in solution.

Determining spectroscopic properties of mSiNC. Absorption spectra and fluorescence emission spectra were obtained with a spectrometer (U-3310; Hitachi) and a spectrofluorometer (F-2500; Hitachi), respectively. The extinction coefficient of the $\mathrm{mSiNC}$ was determined from the absorption spectrum, and the concentration was determined by FCS measurements (described in subsection FCS and determination of the $\mathrm{mSiNC}$ size based on the FCS measurements). The correction for nonfluorescent crystals was made as described in Fig. 5, Table S1, and their related text (in the subsection Each individual $\mathrm{mSiNC}$ neither blinks nor photobleaches).

The quantum yield of the mSiNC was measured by using quinine bisulfate $(Q B)$ as a standard, with excitation at $350 \mathrm{~nm}$; corrected fluorescence spectra of $\mathrm{mSiNC}$ in $50 \mathrm{mM}$ phosphate buffer, $\mathrm{pH} \mathrm{7.0,} \mathrm{and}$ quinine bisulfate in $1 \mathrm{M} \mathrm{H}_{2} \mathrm{SO}_{4}$ were obtained at $25^{\circ} \mathrm{C}$ under exactly the same excitation conditions, for three totally independent preparations of the samples. The optical density at the peak was kept at $<0.1$, and the integrated intensities of the emission spectra, corrected for differences in index of refraction and concentration (Eaton, 1988), were used to calculate the quantum yield $(Q Y)$ according to the equation $Q Y=\left(A b s_{Q B}\right)$ $\left.A b s_{\mathrm{mSiNC}}\right)\left(S_{\mathrm{mSiNC}} / S_{Q B}\right) Q Y_{Q B}$, in which $A b s$ is the absorbance at $350 \mathrm{~nm}$, $S$ is the integrated area under the fluorescence spectrum, and $Q Y_{Q B}$ is 0.546 in $1 \mathrm{M} \mathrm{H}_{2} \mathrm{SO}_{4}$ (Melhuish, 1961). Because corrected fluorescence spectra were used and excitation conditions were exactly matched, the obtained QY value must be accurate.

DLS measurement of the mSiNC size. DLS of mSiNC purified by Superdex 75 gel filtration HPLC was measured using a particle size instrument (Zetasizer Nano ZS 90; Malvern Instruments Limited). The results were analyzed by its software, and the average hydrodynamic diameter ( $z$ average) and polydispersity index were obtained (Koppel, 1972; Thomas, 1987).

FCS and determination of the mSiNC size based on the FCS measurements. The FCS system (ConfoCor 3; Carl Zeiss) consisted of an inverted microscope equipped with a water immersion objective lens $(40 x, 1.2 \mathrm{NA}$; Carl Zeiss), an $\mathrm{Ar}^{+}$laser, an avalanche photodiode, and a digital correlator. All samples were placed in chambered 140-jm-thick cover glasses (Lab-Tek; Thermo Fisher Scientific) and were measured at $25^{\circ} \mathrm{C}$. Samples were excited by the 458-nm line of the laser, and the fluorescence from the detection volume $(0.27 \mathrm{fl})$, separated by a dichroic mirror $(458 \mathrm{~nm})$ and a long-pass filter $(470 \mathrm{~nm})$, was detected.

Fluorescence autocorrelation curves $G(\tau)$ were fitted to a singlecomponent diffusion model (Yoshida et al., 2001; Jin et al., 2007) as follows:

$$
G(\tau)=1+\frac{1}{N}\left(1+\frac{\tau}{\tau_{\text {diffusion }}}\right)^{-1}\left(1+\frac{\tau}{\left(\omega_{z} / \omega_{0}\right)^{2} \tau_{\text {diffusion }}}\right)^{-1 / 2},
$$

in which $N$ is the average number of fluorescent particles in the confocal volume of the lateral radius $\omega_{0}$ and axial radius $\omega_{z \prime}$ and $\tau_{\text {diffusion }}$ is the diffusion time of the fluorescent particles, which is related to the diffusion coefficient $D$ and $\omega_{0}$ by the following equation: $\tau_{\text {diffusion }}=\omega_{0}{ }^{2} / 4 D$.

For spherical particles, $D$ is inversely proportional to its hydrodynamic radius $r$ according to the Stokes-Einstein relationship $\left(D=k_{B} T\right.$ ) $6 \pi \eta r)$, in which $k_{B}$ is the Boltzmann constant, $T$ is the absolute temperature, and $\eta$ is the viscosity of the solution (water $=0.894 \mathrm{cP}$ at $25^{\circ} \mathrm{C}$ ). Thus, the hydrodynamic diameters $d_{m \operatorname{SiNC}}(=2 r)$ of the $\mathrm{mSiNC}$ can be calculated by the following equation:

$$
d_{m \operatorname{SiNC}}=\left(\frac{k_{B} T}{3 \pi \eta}\right)\left(\frac{\tau_{\text {diffusion }}(m \operatorname{SiNC})}{D(\text { standard }) \tau_{\text {diffusion }}(\text { standard })}\right),
$$

in which $D$ (standard) is the diffusion coefficient of a standard dye, and $\tau_{\text {diffusion }}\left(\mathrm{mSiNC}\right.$ ) and $\tau_{\text {diffusion }}$ (standard) are the diffusion times of the $\mathrm{mSiNC}$ and the standard dye, respectively. As the standard dye, rhodamine $6 G$ $\left(2.8 \times 10^{-6} \mathrm{~cm}^{2} / \mathrm{s}\right.$; Rigler et al., 1993), monomeric GFP, and Qdot655 were used. Samples were measured for 10 runs of $20 \mathrm{~s}$ each at $25^{\circ} \mathrm{C}$.

TEM for determining the size distribution of mSiNC. Purified $\mathrm{mSiNC}$ were mounted on polyvinyl Formvar-coated grids (Pelco) and imaged with a transmission electron microscope (JEM-1400 [JEOL] and Tecnai G2 Spirit $[\mathrm{FEI}])$. The sizes of the individual $\mathrm{mSiNC}$ particles were determined using ImageJ software (Threshold: Maximum entropy and Analyze particles; National Institutes of Health).

Determination of the amount of mercaptosilane bound to, and the extinction coefficient of, mSiNC. A suspension of $\sim 3.2$-nm-diameter $\mathrm{mSiNC}$, purified by gel filtration HPLC, was dialyzed against $10 \mathrm{mM}\left(\mathrm{NH}_{4}\right)_{2} \mathrm{CO}_{3}$, using 3,500 molecular weight cut-off dialysis cassettes (Slide-A-Lyzer). The resulting solution was freeze dried to remove water, $\mathrm{NH}_{3}$, and $\mathrm{CO}_{2}$, and the remaining $\mathrm{mSiNCs}$ were weighed with a 100-ng scale balance (SE2; Sartorius).

The amount of mercaptosilane bound to $\mathrm{mSiNC}$ was determined by quantifying the thiol group of 3-MPTS on the particle, by a reaction with 4,4'-dithiodipyridine (Grassetti and Murray, 1967), using 1-64 NM 3-MPTS dissolved in purified water as standard solutions. A $100-\mu$ sample was mixed with $40 \mathrm{\mu l}$ of $50-\mathrm{mM}$ EDTA, $\mathrm{pH} 6.0$, and then incubated at $30^{\circ} \mathrm{C}$ for $30 \mathrm{~min}$. We then added $10 \mu \mathrm{l}$ of 5-mM 4,4'-dithiodipyridine and incubated at $30^{\circ} \mathrm{C}$ for $15 \mathrm{~min}$. The reaction was monitored by the optical density at $324 \mathrm{~nm}$, and the mole amount of 3-MPTS (molecular weight $=155$ ) bound to $\mathrm{mSiNC}$ was determined.

Next, by subtracting the weight of 3-MPTS bound to the mSiNC from the weight of $\mathrm{mSiNC}$ measured by a sensitive balance (as described in the first paragraph in this subsection), the weight of the $\mathrm{mSiNC}$ without the coating (i.e., SiNC) was determined. To obtain the molar amounts of $\mathrm{mSiNC}$, the molecular weight of 3.2-nm SiNC (the diameter evaluated by EM) was evaluated in the following way. Because a 1-nm-diameter $\mathrm{SiNC}$ has a molecular weight of $836\left(\mathrm{Si}_{29} \mathrm{H}_{24}\right.$; Belomoin et al., 2002), we estimated the molecular weight of the 3.2-nm SiNC to be 26,853.4 $\left(\mathrm{Si}_{950.3} \mathrm{H}_{245.8}\right)$, based on the 3D packing of silicon atoms $\left([3.2 \mathrm{~nm} / 1 \mathrm{~nm}]^{3} \times\right.$ $29=950.3 \mathrm{Si}$ atoms) and the $2 \mathrm{D}$ packing of hydrogen atoms on the surface $\left([3.2 \mathrm{~nm} / 1 \mathrm{~nm}]^{2} \times 24=245.8 \mathrm{H}\right.$ atoms; the number of hydroxyl groups formed on the surface was not assessed). Using this molecular weight, the mole amounts of 3.2-nm SiNC in given solutions (equal to the mole amounts of $\mathrm{mSiNC}$ in given solutions) were obtained.

These measurements allowed us to determine that $51.2 \pm 19.4$ (mean \pm SD, $n=3$ ) 3-MPTS molecules were bound to each mSiNC particle, giving a mean molecular weight of $\mathrm{mSiNC}$ of 36,083.6 $(26,853.4+$ $155 \times 51.2$ ). The extinction coefficient of $\mathrm{mSiNC}$ was determined from the absorption spectrum of a given solution and the mole amount of $\mathrm{mSiNC}$ in the solution.

Long-term storage mSiNCs and the preparation and observation of mSiNC-If in aqueous solutions during a day. For long-term storage of the $\mathrm{mSiNCs}$, they were stored in dehydrated benzene at $4^{\circ} \mathrm{C}$ for $\leq 60 \mathrm{~d}$ (Fig. S1). However, in aqueous solutions, the fluorescence intensity was reduced much faster. The fluorescence intensity of $\mathrm{mSiNC}$ decreases with an exponential decay time of $42 \pm 2.8 \mathrm{~h}$ in PBS at $37^{\circ} \mathrm{C}$, as described in Fig. S2. In a typical schedule of $\mathrm{mSiNC}$ conjugation to Tf and single-molecule observations of mSiNC bound to the cell surface (after obtaining the mSiNCs or after the change of the solvent from benzene to an aqueous buffer), it takes $\sim 2 \mathrm{~h}$ for Tf conjugation and mSiNC-Tf purification and another $4 \mathrm{~h}$ for the single-molecule tracking observations for the day. Therefore, we examined the fluorescence spectrum, gel filtration HPLC elution pattern, and the FCS time series and autocorrelation function after incubation of mSiNC-Tf in 
PBS at $37^{\circ} \mathrm{C}$ for $6 \mathrm{~h}$. Based on these observations, we recommend that $\mathrm{mSiNCs}$ be kept in dehydrated benzene at $4^{\circ} \mathrm{C}$ for long-term storage and that on the day of the actual cell biology experiment, the benzene be evaporated by a flow of nitrogen gas flow and then aqueous buffer be added to dried $\mathrm{mSiNCs}$.

\section{Single fluorescent molecule imaging/tracking of mSiNC and mSiNC-labeled If}

Single-color single fluorescent molecule tracking, using TIRF microscopy. Homebuilt objective lens-type TIRF microscopes were constructed on the basis of inverted microscopes (IX70 [Olympus] and TE2000 [Nikon]), equipped with oil immersion objective lenses (Plan Apochromat 60x, NA 1.45 [Olympus]; Plan Apochromat 100x NA 1.49 [Nikon]) and 495-nm dichroic mirrors (FF495-Di02; Semrock), as described previously (Murakoshi et al., 2004; Koyama-Honda et al., 2005; Umemura et al., 2008). The 405- and 440-nm lines of solid-state lasers (BCL-050-405 and BCL-040440; CrystaLaser) were used to excite the mSiNC, Qdot655, and Cy3 samples for the Olympus- and Nikon-based stations, respectively. Stray excitation light was blocked with a long-pass filter (E590LPv2; Chroma Technology Corp.). Fluorescence images were detected by an image intensifier (C8600-03; Hamamatsu Photonics) lens-coupled to an electron bombardment charge-coupled device (CCD) camera (C7190-23; Hamamatsu Photonics), and the obtained images were recorded on a digital videotape (PDV-184ME; Sony). Each individual fluorescent spot was identified and analyzed by using a homemade computer program as described previously (Fujiwara et al., 2002). All of the observations were made at $37^{\circ} \mathrm{C}$ using Hanks' buffered salt solution.

The TIRF apparatus is not required for the observations of single $\mathrm{mSiNC}$ particles, and a typical epifluorescence apparatus is sufficient for single $\mathrm{mSiNC}$ particle tracking at video rate. However, because our focus in this research is single fluorescent molecule (particle) tracking and because TIRF illumination is now a standard in laboratories conducting single fluorescent molecule tracking, we also show the data obtained by TIRF illumination in this study.

Two-color (two molecular species) single fluorescent molecule tracking by TIRF microscopy. An objective lens-type TIRF microscope was constructed on an inverted microscope (TE2000; Nikon), equipped with an oil immersion objective lens (Plan Apochromat 100x, NA 1.49; Nikon) and 495-nm dichroic mirrors (FF495-Di02; Semrock), as described previously (Murakoshi et al., 2004; Koyama-Honda et al., 2005). The 440 (BCL-040440; CrystaLaser)- and 488 (Excelsior One-488-100; Spectra-Physics)-nm lines of solid-state lasers were used to excite the Alexa Fluor $488-\mathrm{mSiNC}$ samples. The fluorescence images of $\mathrm{mSiNC}$ and Alexa Fluor 488 were separated by a dichroic mirror (FF562-Di02; Semrock) and projected into two detection arms with a long-pass filter (>590 nm for mSiNC; E590LPv2) and a band-pass filter (520/35 nm for Alexa Fluor 488; Semrock). An image-intensifier (C8600-03) lens coupled to an electron bombardment CCD camera (C7190-23) was used in each arm. Fluorescent images were recorded and analyzed as described in the previous section.

Fluorescence intensity measurements. Fluorescence images were digitized frame by frame with a homemade computer program (Fujiwara et al., 2002). The signal intensity of $530 \times 530-n m$ areas (8-bit images in $10 \times$ 10 pixels), each containing a single spot, was measured. The background intensity of an adjacent area was subtracted for each spot.

Detection of transient confinement zones and time (area)-dependent changes of diffusion coefficient. To detect nonrandom confinement in a zone, or a transient confinement zone, we looked for periods in which the protein remains in a membrane region for a duration considerably longer than a Brownian diffusant would stay in an equally sized region, based on the theory and program developed by Simson et al. (1995). For every point within a trajectory, the probability level $L$, which represents the probability that the particle is confined to a region longer than a random diffusant would remain, is assigned. Plotting $L$ as a function of time yields a probability profile of the trajectory, as shown in Fig. S5 C. High values in the profile denote periods when the probability that the particle is confined to a region is high. A period of confined diffusion is defined by the position where the profile rises above a critical threshold level $L c$ for a duration longer than tc (Fig. S5 C). The settings $L c=2.20, t c=0.333 \mathrm{~s}$, and $S m=$ $1.00 \mathrm{~s}$ were chosen based on the results of 500 simulated Brownian trajectories of 500 steps. Under these conditions, the threshold $L c=2.20$ is $<2 \%$ likely to have a random origin.

The SD $r^{2}\left(t_{i}\right)$ during a time interval $\left[t_{i}, t_{i+1}\right]$ is defined as $r^{2}\left(t_{i}\right)=\left(x\left(t_{i+1}\right)-\right.$ $\left.x\left(t_{i}\right)\right)^{2}+\left(y\left(t_{i+1}\right)-y\left(t_{i}\right)\right)^{2}$, in which $\left(x\left(t_{i}\right), y\left(t_{i}\right)\right)$ is the position of the TfR-mSiNCTf at time $t_{i}$ in the trajectory. Here, $t_{i}\left(t_{i}=i \times t_{b}\right)$ is an integer multiple of the bin time $t_{b}(33 \mathrm{~ms})$. A CSD, $C_{R}(t)$, is obtained by the cumulative sum of SD from time 0 to $t\left(=n \times t_{b}\right)$, such as

$$
C_{R}(t)=\sum_{i=0}^{n} r^{2}\left(t_{i}\right)
$$

$S D$ and $C S D$ can be related to the diffusion coefficient $D$, as $S D=$ $4 D t_{b}$ and $C S D=4 D t$. SD would fluctuate greatly as a result of the lack of averaging, but the plot of CSD versus time would fluctuate much less, and $D$ obtained from this slope would reflect the long-term diffusion coefficient. Therefore, the long-term diffusion coefficient for the specific time period could be directly obtained from this plot (Chung et al., 2010).

The plot of CSD versus time obtained from a typical trajectory is shown in Fig. 8 B (top). The changes in diffusivity could be identified in the plot, as the time intervals with different slopes. In this analysis, we imposed $5 \mathrm{~s}$ as the minimum duration for a given diffusivity state, within which any possible change in diffusion dynamics is ignored because a small number of plots leads to increasing uncertainty in the observations. The diffusion coefficient was estimated from the linear regression of the CSD- $t$ $(t=3$ s) plot.

The effective diffusion coefficient in the time scale of $100 \mathrm{~ms}\left(D_{100 \mathrm{~ms}}\right.$; Fig. 7 E) for each fluorescent spot was obtained as described previously (Kusumi et al., 1993; Fujiwara et al., 2002). In brief, for each particle's trajectory, the mean $S D(M S D),<\Delta r(\Delta t)^{2}>$, for every time interval $(\Delta t=n \delta t)$ was calculated according to the formula

$M S D(n \delta t)=\frac{1}{N-1-n} \sum_{i=1}^{N-1-n}\left\{[x(j \delta t+n \delta t)-x(j \delta t)]^{2}+[y(j \delta t+n \delta t)-y(j \delta t)]^{2}\right\}$,

in which $\delta t$ is the video frame time $(1 / 30 \mathrm{~s})$, and $(x(j \delta t+n \delta t), y(j \delta t+n \delta t))$ describes the particle position after a time interval $\Delta t_{n}=n \delta t$ after starting at position $(x(j \delta t), y(j \delta t), N$ is the total number of frames in the video recording sequence, $n$ and $j$ are positive integers, and $n$ determines the time increment. $D_{100 \mathrm{~ms}}$, corresponding to $D_{2-4}$ defined in Kusumi et al. (1993), was determined by linearly fitting the plot of $M S D$ versus $\Delta t$ between the second and fourth points.

Preparation of Alexa Fluor 488-labeled mSiNC (Alexa Fluor 488-mSiNC) and determination of the fraction of $\mathrm{mSiNCs}$ that are actually fluorescent. The Alexa Fluor 488-labeled $\mathrm{mSiNC}$ was basically prepared essentially as described previously (Fujiwara et al., 2002; Umemura et al., 2008). In brief, $10 \mu$ Alexa Fluor 488 C5-maleimide dye $(0.2 \mathrm{mM}$; Molecular Probes) in dimethylformamide was added to $200 \mu \mathrm{mSiNC}(\sim 1 \mu \mathrm{M})$ in $20 \mathrm{mM}$ phosphate buffer, $\mathrm{pH} 7.0$, and the mixture was incubated at room temperature for $60 \mathrm{~min}$. Alexa Fluor $488-\mathrm{mSiNC}$ (+unreacted $\mathrm{mSiNC}$ ) was separated from the free Alexa Fluor 488 dye by Superdex 75 gel filtration HPLC equilibrated with $50 \mathrm{mM}$ phosphate buffer, $\mathrm{pH}$ 7.0. The dye $/ \mathrm{mSiNC}$ ratio of the samples used for two-color single fluorescent molecule tracking was $0.35 \pm 0.031$ (mean $\pm S D ; n=3$ ), which was determined using their respective molar extinction coefficient of 53,000 and $71,000 \mathrm{M}^{-1} \mathrm{~cm}^{-1}$ (at 400 and $495 \mathrm{~nm}$, respectively [Table S1]; there is virtually no overlap of the spectra of $\mathrm{mSiNC}$ and Alexa Fluor 488 at these wavelengths).

Using Alexa Fluor 488-mSiNC (the mSiNCs labeled with Alexa Fluor 488 at a mean Alexa Fluor/mSiNC molar ratio of 0.35), we examined the fractions of fluorescent and nonfluorescent mSiNCs. For this examination, the fluorescence signals emitted from Alexa Fluor 488 and $\mathrm{mSiNCs}$, each excited with a 488- or 440-nm laser, respectively, were observed. First, all of the Alexa Fluor spots in the view field were identified (because of possible FRET from Alexa Fluor 488 to mSiNCs [although this FRET efficiency appeared to be very low because single-particle spots were hardly detected in $\mathrm{mSiNC}$ channel with the excitation at $488 \mathrm{~nm}$ ], some of the Alexa Fluor molecules might not have been detected in the Alexa Fluor 488 channel, but this will not affect the final result because we made further examination only for the detected Alexa Fluor spots). Then, each identified Alexa Fluor spot was examined whether it was colocalized by an $\mathrm{mSiNC}$ spot (Koyama-Honda et al., 2005; No single-particle spots were detected in the Alexa Fluor 488 channel with the 440-nm excitation for $\mathrm{mSiNC}$, indicating very low efficiency of FRET from $\mathrm{mSiNC}$ to Alexa Fluor488). This way, the fraction of Alexa Fluor 488 spots colocalized by fluorescent $\mathrm{mSiNC}$ spots was determined. The numbers of Alexa Fluor spots that were colocalized or noncolocalized by the mSiNCs spots were counted (the Alexa Fluor $488 / \mathrm{mSiNC}$ ratio of 0.35 did not affect further 
calculations of the percentage of dark mSiNC particles because we first detected Alexa Fluor 488 spots and then examined for each Alexa Fluor 488 spot whether it is colocalized by the mSiNC fluorescence spot; Fig. 5). The result showed that $38.4 \pm 2.6 \%$ (mean \pm SD) of the SiNCs were actually fluorescent (for six independently prepared specimens; examining 245 Alexa Fluor 488 spots in total), indicating much improvement from the previously reported value of $2.8 \%$, although the improvement mechanism is not clear.

Preparation of Cy3-labeled Tf (Cy3-Tf). The Cy3-labeled Tf was basically prepared as described previously (Fuijwara et al., 2002; Umemura et al., 2008). To outline the process briefly, to $200 \mu$ Tf $(10 \mu \mathrm{M})$ in PBS, $\mathrm{pH} 7.2,10 \mu \mathrm{l} \mathrm{Cy} 3$ monoreactive dye (1.6 mM; GE Healthcare) in dimethylformamide was added, and the mixture was incubated at room temperature for $60 \mathrm{~min}$. Cy3-Tf (+unlabeled Tf) was separated from the free Cy3 dye by chromatography on a Sephadex G-25 column (GE Healthcare) equilibrated with PBS. The dye/protein ratio of the samples used for single fluorescent molecule tracking was $0.39 \pm 0.062$ (mean $\pm S D ; n=4$ ), using the molar extinction coefficients of $150,000 \mathrm{M}^{-1} \mathrm{~cm}^{-1}$ (at $552 \mathrm{~nm}$ ) for Cy3 (Table S1) and 83,360 $\mathrm{M}^{-1} \mathrm{~cm}^{-1}$ (at $280 \mathrm{~nm}$ ) for Tf (West et al., 2000).

Cell preparation for fluorescence microscopy. To outline the process briefly, the NRK fibroblast cell line was grown in Ham's F12 medium supplemented with antibiotics and 10\% FBS. CEF cells, purchased from ATCC, were grown in DMEM medium supplemented with antibiotics, 5\% tryptose phosphate broth, and 5\% FBS. Cells were plated on 12-mm $\varphi$ coverslip-based dishes (IWAKI) 2 d before being observed. Shortly before observation, the cells were preincubated in Hanks' buffered salt solution at $37^{\circ} \mathrm{C}$ for $15 \mathrm{~min}$ to deplete Tf from the cell surface, and then, mSiNC-Tf or Cy3-Tf, eluted from the column, was added to a final concentration of 0.5-5 nM.

As shown in Fig. 7, the cells internalize TfRs (labeled with mSiNC-Tf) at the same rate, even after illumination with a 440 -nm laser line, for $\geq 5 \mathrm{~min}$. The cellular morphology did not change appreciably during this period. Therefore, although due caution is certainly required, our observation conditions of exciting mSiNC on the cell surface with a 440-nm laser line would be acceptable for the types of experiments described in this study.

Determining the observation durations for single particles/molecules. The observation duration for a single molecule (particle) was determined in terms of the number of observation frames (images), rather than in terms of the total observation time. Because the overall observation duration expressed in terms of time can be prolonged by time lapsing, the number of observed frames, and not the observed time period, is the critical parameter.

Generally, in single-molecule (particle) tracking, even if some frames miss the signal from the tracked single molecule, researchers tend to connect the gap-separated trajectories into a single longer trajectory, and sometimes, the gap lengths are very long or undescribed in the study (Table S2; Bats et al., 2007; Ehrensperger et al., 2007; Heine et al., 2008). In the present research, we were highly restrictive in performing such reconnections. Namely, we did not make any reconnections over the gap. If there was a single frame that missed the detectable fluorescence signal from the particle, our tracking finished right at that moment. Therefore, the maximal gap for reconnection is 0 here.

Even under these very strict conditions, the observed number of frames for a single mSiNC particle achieved in this work is much longer than that accomplished previously (with less restrictive reconnection conditions). Because we were able to observe the particle for up to $120 \mathrm{~s}$ at video rate $(30 \mathrm{~Hz})$, our total number of observation frames is 3,600 . This is about twice as long as the longest observation frames ever made by QDs (with the use of reconnection; Heine et al., 2008).

The number of observable consecutive frames using $\mathrm{mSiNC}$ was no longer limited by blinking or photobleaching. The limitation was found to be given by the following three factors. (1) The membrane proteins experience the uneven terrain of the plasma membrane, blurring the particle's image. (2) TfRs are internalized quickly. (3) Long-term instability of focus becomes prominent after $60 \mathrm{~s}$ of observation at $37^{\circ} \mathrm{C}$.

In addition, in the previous studies that claim long observation times for single molecules (for either organic dyes or QDs), the experiments are often conducted under at least one of the following conditions. (1) Deoxygenation, using glucose oxidase-catalase system, oxyrase, or simple replacement of air with nitrogen or argon (Aitken et al., 2008), which could make the observed cells very sick. (2) Reductive conditions, using high concentrations of reducing reagents such as $\beta$-mercaptoethanol (Vogelsang et al., 2008) and deoxygenation, which could drastically alter the cells' metabolism. (3) Arbitrary selection of the incidentally occurring long trajectories.

Under the reductive conditions in the cell, the QDs blink less frequently, but they could induce many adversary effects on the cell, and yet suppression of blinking would not be complete. We are not aware of any methods that can suppress the QD blinking to the level at which singlemolecule tracking in/on living cells could be useful. Therefore, we conclude that the $\mathrm{mSiNC}$ described in this paper would allow researchers to perform single-molecule (particle) tracking experiments under conditions that are much more favorable for live cells and to obtain trajectories with far more steps (without reconnection) than are possible with other probes.

\section{Observation of GFP-hTfR and mSiNC-Tf in/on the live CEF cell plasma membrane}

Plasmid construction. The cDNA encoding full-length, wild-type hTfR, with the linker sequence $5^{\prime}$-AATTCT-hTfR-3' added to the $5^{\prime}$ end, was cloned into the pcDNA3+ vector (Invitrogen). A monomeric GFP mutant (A206K mutation) cDNA, with the linker sequence 5'-TCCGGACTCAGATCTCGAGCTCAAGCTTCG-3' added to the 5' end, was then placed in this vector, yielding the GFP-hTfR expression vector (note that the $\mathrm{N}$ terminus of TfR is located in the cytoplasm).

Epifluorescence microscopy. Epifluorescence was constructed on an inverted microscope (IX70), equipped with an oil immersion objective lens (U Plan Fluar 60x, NA 1.25; Olympus). mSiNC-Tf (350/50 nm excitation, $>590 \mathrm{~nm}$ emission, and dichroic mirror [400DCLP; Chroma Technology Corp.]) and GFP-hTfR (470/40 nm excitation, 535/50 nm emission, and dichroic mirror [505DCLP; Chroma Technology Corp.]) images were detected by a digital CCD camera (Quantix; Photometrics) and analyzed using MetaVue image analysis software version 7.1.5.0 (Molecular Devices).

Cell culture and cDNA transfection for epifluorescence microscopy. CEF cells were maintained in DMEM medium supplemented with antibiotics, $5 \%$ tryptose phosphate broth, and 5\% FBS and were transfected with the cDNAs using Lipofectamine Plus (Invitrogen) according to the manufacturer's recommendations. For fluorescence microscopy, cells were plated on 12-mm $\varphi$ coverslip-based dishes and were used in $2 \mathrm{~d}$. Shortly before observation, the cells were preincubated in Hanks' buffered salt solution at $37^{\circ} \mathrm{C}$ for $15 \mathrm{~min}$ to deplete Tf from the cell surface, and then, mSiNC-Tf, eluted from the column, was added to a final concentration of 10-100 nM.

\section{Online supplemental material}

Fig. S1 shows that the fluorescence properties of the $\mathrm{mSiNC}$ are not affected during storage for $60 \mathrm{~d}$ in dehydrated benzene. Fig. S2 shows time-dependent changes of the integrated fluorescence intensity lexcited at $350 \mathrm{~nm}$ ) of the mSiNC-Tf in $50 \mathrm{mM}$ phosphate buffer, pH 7.0. Fig. S3 shows that mSiNC neither blinked nor photobleached, whereas QDs frequently blinked and gradually photobleached. Fig. S4 shows that fluorescence intensity of mSiNC is linearly increased with an increase of the excitation laser intensity at least up to $0.68 \mu \mathrm{W} /{\mu \mathrm{m}^{2}}^{2}$, twice the intensity generally used in the present research. Fig. S5 shows specific binding of mSiNC-Tf (human Tf) to TfR and a typical trajectory of this molecular complex (mSiNC-Tf and TfR) on the NRK cell surface including a period of temporary confinement followed by a (probable) internalization process. Table S1 summarizes spectral properties of $\mathrm{mSiNC}$, commercial organic dyes, and Qdot655. Table S2 lists the total number of consecutive images (frames) acquired for single particles of Qdot655, Cy3, and $\mathrm{mSiNC}$ before the particle became untrackable and their maximal gap in reconnection. Video 1 shows fluorescence images of single mSiNCs and single QDs. Video 2 shows that individual TfR molecules, each tagged with an mSiNC-Tf, undergo rapid apparent simple Brownian diffusion in the plasma membrane of a live NRK cell. Video 3 shows the result of direct visualization of internalization process of a TfR molecule tagged with mSiNC-Tf in the plasma membrane of a live NRK cell. Online supplemental material is available at http://www.jcb.org/cgi/content/full/jcb.201301053/DC1

We thank Koji Kanemasa for help with figure preparation.

This work was supported in part by World Premier International Research Center Initiative of the Ministry of Education, Culture, Sports, Science and Technology, the Program for Promotion of Fundamental Studies in Health Sciences of the National Institute of Biomedical Innovation (N. Morone), and Grants-in-Aid from the Ministry of Education, Culture, Sports, Science and Technology (A. Kusumi).

Author contributions: $\mathrm{H}$. Nishimura performed a large majority of the experiments reported here. K.P. Ritchie and M. Goto developed a new SiNC preparation method at the initial stages of this work. N. Morone performed TEM, and H. Nishimura and H. Sugimura performed FTIR observations. Y. Nakano and I. Sase designed and produced the autofocus system for the microscope. H. Nishimura, R.S. Kasai, and T.K. Fujiwara performed single-molecule imaging. A. Yoshimura, K. Tanaka, I. Sase, Y. Nakano, and A. Kusumi evaluated 
and discussed data. H. Nishimura and A. Kusumi wrote the manuscript, and K. Ritchie, A. Yoshimura, Y. Nakano, K. Tanaka, T.K. Fujiwara, and A. Kusumi formulated the project.

Submitted: 15 January 2013

Accepted: 6 August 2013

\section{References}

Aitken, C.E., R.A. Marshall, and J.D. Puglisi. 2008. An oxygen scavenging system for improvement of dye stability in single-molecule fluorescence experiments. Biophys. J. 94:1826-1835. http://dx.doi.org/10.1529/ biophysj.107.117689

Akcakir, O., J. Therrien, G. Belomoin, N. Barry, J.D. Muller, E. Gratton, and M. Nayfeh. 2000. Detection of luminescent single ultrasmall silicon nanoparticles using fluctuation correlation spectroscopy. Appl. Phys. Lett. 76:1857-1859. http://dx.doi.org/10.1063/1.126191

Altman, R.B., D.S. Terry, Z. Zhou, Q. Zheng, P. Geggier, R.A. Kolster, Y. Zhao, J.A. Javitch, J.D. Warren, and S.C. Blanchard. 2012a. Cyanine fluorophore derivatives with enhanced photostability. Nat. Methods. 9:68-71. http://dx.doi.org/10.1038/nmeth.1774

Altman, R.B., Q. Zheng, Z. Zhou, D.S. Terry, J.D. Warren, and S.C. Blanchard. 2012b. Enhanced photostability of cyanine fluorophores across the visible spectrum. Nat. Methods. 9:428-429. http://dx.doi.org/10.1038/ nmeth. 1988

Bats, C., L. Groc, and D. Choquet. 2007. The interaction between Stargazin and PSD-95 regulates AMPA receptor surface trafficking. Neuron. 53:719-734. http://dx.doi.org/10.1016/j.neuron.2007.01.030

Belomoin, G., J. Therrien, A. Smith, S. Rao, R. Twesten, S. Chaieb, M.H. Nayfeh, L. Wagner, and L. Mitas. 2002. Observation of a magic discrete family of ultrabright Si nanoparticles. Appl. Phys. Lett. 80:841-843. http://dx.doi .org/10.1063/1.1435802

Buschmann, V., K.D. Weston, and M. Sauer. 2003. Spectroscopic study and evaluation of red-absorbing fluorescent dyes. Bioconjug. Chem. 14:195-204. http://dx.doi.org/10.1021/bc025600x

Canham, L.T. 1990. Silicon quantum wire array fabrication by electrochemical and chemical dissolution of wafers. Appl. Phys. Lett. 57:1046-1048. http://dx.doi.org/10.1063/1.103561

Chadda, R., M.T. Howes, S.J. Plowman, J.F. Hancock, R.G. Parton, and S. Mayor. 2007. Cholesterol-sensitive Cdc42 activation regulates actin polymerization for endocytosis via the GEEC pathway. Traffic. 8:702-717. http://dx.doi.org/10.1111/j.1600-0854.2007.00565.x

Chen, Y., J. Vela, H. Htoon, J.L. Casson, D J. Werder, D.A. Bussian, V.I. Klimov, and J.A. Hollingsworth. 2008. "Giant" multishell CdSe nanocrystal quantum dots with suppressed blinking. J. Am. Chem. Soc. 130:5026-5027. http://dx.doi.org/10.1021/ja711379k

Choi, J., N.S. Wang, and V. Reipa. 2008. Conjugation of the photoluminescent silicon nanoparticles to streptavidin. Bioconjug. Chem. 19:680-685. http://dx.doi.org/10.1021/bc700373y

Chung, I., R. Akita, R. Vandlen, D. Toomre, J. Schlessinger, and I. Mellman. 2010. Spatial control of EGF receptor activation by reversible dimerization on living cells. Nature. 464:783-787. http://dx.doi.org/10.1038/ nature 08827

Cognet, L., L. Groc, B. Lounis, and D. Choquet. 2006. Multiple routes for glutamate receptor trafficking: surface diffusion and membrane traffic cooperate to bring receptors to synapses. Sci. STKE. 2006:pe13.

Colton, C.K., K.A. Smith, E.W. Merrill, and P.C. Farrell. 1971. Permeability studies with cellulosic membranes. J. Biomed. Mater. Res. 5:459-488. http://dx.doi.org/10.1002/jbm.820050504

Credo, G.M., M.D. Mason, and S.K. Buratto. 1999. External quantum efficiency of single porous silicon nanoparticles. Appl. Phys. Lett. 74:1978-1980. http://dx.doi.org/10.1063/1.123719

de Boer, W.D., D. Timmerman, K. Dohnalová, I.N. Yassievich, H. Zhang, W.J. Buma, and T. Gregorkiewicz. 2010. Red spectral shift and enhanced quantum efficiency in phonon-free photoluminescence from silicon nanocrystals. Nat. Nanotechnol. 5:878-884. http://dx.doi.org/10.1038/nnano .2010 .236

Dickson, R.M., A.B. Cubitt, R.Y. Tsien, and W.E. Moerner. 1997. On/off blinking and switching behaviour of single molecules of green fluorescent protein. Nature. 388:355-358. http://dx.doi.org/10.1038/41048

Eaton, D.F. 1988. International union of pure and applied chemistry organic chemistry division commission on photochemistry. Reference materials for fluorescence measurement. J. Photochem. Photobiol. B. 2:523-531. http://dx.doi.org/10.1016/1011-1344(88)85081-4

Ehrensperger, M.V., C. Hanus, C. Vannier, A. Triller, and M. Dahan. 2007. Multiple association states between glycine receptors and gephyrin identified by SPT analysis. Biophys. J. 92:3706-3718. http://dx.doi.org/ 10.1529/biophysj.106.095596

Erogbogbo, F., K.T. Yong, I. Roy, G. Xu, P.N. Prasad, and M.T. Swihart. 2008 Biocompatible luminescent silicon quantum dots for imaging of cancer cells. ACS Nano. 2:873-878. http://dx.doi.org/10.1021/nn700319z

Erogbogbo, F., C.A. Tien, C.W. Chang, K.T. Yong, W.C. Law, H. Ding, I. Roy, M.T. Swihart, and P.N. Prasad. 2011a. Bioconjugation of luminescent silicon quantum dots for selective uptake by cancer cells. Bioconjug. Chem. 22:1081-1088. http://dx.doi.org/10.1021/bc100552p

Erogbogbo, F., K.T. Yong, I. Roy, R. Hu, W.C. Law, W. Zhao, H. Ding, F. Wu, R. Kumar, M.T. Swihart, and P.N. Prasad. 2011b. In vivo targeted cancer imaging, sentinel lymph node mapping and multi-channel imaging with biocompatible silicon nanocrystals. ACS Nano. 5:413-423. http://dx.doi .org/10.1021/nn1018945

Fujiwara, T., K. Ritchie, H. Murakoshi, K. Jacobson, and A. Kusumi. 2002. Phospholipids undergo hop diffusion in compartmentalized cell membrane. J. Cell Biol. 157:1071-1081. http://dx.doi.org/10.1083/jcb.200202050

Gelloz, B., H. Sano, R. Boukherroub, D.D.M. Wayner, D.J. Lockwood, and N. Koshida. 2003. Stabilization of porous silicon electroluminescence by surface passivation with controlled covalent bonds. Appl. Phys. Lett. 83:2342-2344. http://dx.doi.org/10.1063/1.1613812

Germain, R.N., E.A. Robey, and M.D. Cahalan. 2012. A decade of imaging cellular motility and interaction dynamics in the immune system. Science. 336:1676-1681. http://dx.doi.org/10.1126/science.1221063

Grassetti, D.R., and J.F. Murray Jr. 1967. Determination of sulfhydryl groups with 2,2'- or 4,4'-dithiodipyridine. Arch. Biochem. Biophys. 119:41-49. http://dx.doi.org/10.1016/0003-9861(67)90426-2

Ha, T., and P. Tinnefeld. 2012. Photophysics of fluorescent probes for singlemolecule biophysics and super-resolution imaging. Annu. Rev. Phys. Chem. 63:595-617. http://dx.doi.org/10.1146/annurev-physchem-032210103340

Heine, M., L. Groc, R. Frischknecht, J.C. Béique, B. Lounis, G. Rumbaugh, R.L. Huganir, L. Cognet, and D. Choquet. 2008. Surface mobility of postsynaptic AMPARs tunes synaptic transmission. Science. 320:201-205. http://dx.doi.org/10.1126/science.1152089

Howarth, M., W. Liu, S. Puthenveetil, Y. Zheng, L.F. Marshall, M.M. Schmidt, K.D. Wittrup, M.G. Bawendi, and A.Y. Ting. 2008. Monovalent, reducedsize quantum dots for imaging receptors on living cells. Nat. Methods. 5:397-399. http://dx.doi.org/10.1038/nmeth.1206

Iino, R., I. Koyama, and A. Kusumi. 2001. Single molecule imaging of green fluorescent proteins in living cells: E-cadherin forms oligomers on the free cell surface. Biophys. J. 80:2667-2677. http://dx.doi.org/10.1016/ S0006-3495(01)76236-4

Jin, T., F. Fujii, E. Yamada, Y. Nodasaka, and M. Kinjo. 2007. Preparation and characterization of thiacalix[4]arene coated water-soluble $\mathrm{CdSe} /$ $\mathrm{ZnS}$ quantum dots as a fluorescent probe for $\mathrm{Cu}^{2+}$ ions. Comb. Chem. High Throughput Screen. 10:473-479. http://dx.doi.org/10.2174/ 138620707781996466

Jing, S.Q., T. Spencer, K. Miller, C. Hopkins, and I.S. Trowbridge. 1990. Role of the human transferrin receptor cytoplasmic domain in endocytosis: localization of a specific signal sequence for internalization. J. Cell Biol. 110:283-294. http://dx.doi.org/10.1083/jcb.110.2.283

Kasai, R.S., K.G.N. Suzuki, E.R. Prossnitz, I. Koyama-Honda, C. Nakada, T.K. Fujiwara, and A. Kusumi. 2011. Full characterization of GPCR monomer-dimer dynamic equilibrium by single molecule imaging. J. Cell Biol. 192:463-480. http://dx.doi.org/10.1083/jcb.201009128

Koppel, D.E. 1972. Analysis of macromolecular polydispersity in intensity correlation spectroscopy: The method of cumulants. J. Chem. Phys. 57: 4814-4820. http://dx.doi.org/10.1063/1.1678153

Koyama-Honda, I., K. Ritchie, T. Fujiwara, R. Iino, H. Murakoshi, R.S. Kasai, and A. Kusumi. 2005. Fluorescence imaging for monitoring the colocalization of two single molecules in living cells. Biophys. J. 88:21262136. http://dx.doi.org/10.1529/biophysj.104.048967

Kusumi, A., Y. Sako, and M. Yamamoto. 1993. Confined lateral diffusion of membrane receptors as studied by single particle tracking (nanovid microscopy). Effects of calcium-induced differentiation in cultured epithelial cells. Biophys. J. 65:2021-2040. http://dx.doi.org/10.1016/S00063495(93)81253-0

Kusumi, A., C. Nakada, K. Ritchie, K. Murase, K. Suzuki, H. Murakoshi, R.S. Kasai, J. Kondo, and T. Fujiwara. 2005. Paradigm shift of the plasma membrane concept from the two-dimensional continuum fluid to the partitioned fluid: high-speed single-molecule tracking of membrane molecules. Annu. Rev. Biophys. Biomol. Struct. 34:351-378. http://dx.doi .org/10.1146/annurev.biophys.34.040204.144637

Li, X., Y. He, and M.T. Swihart. 2004. Surface functionalization of silicon nanoparticles produced by laser-driven pyrolysis of silane followed by HF- $\mathrm{HNO}_{3}$ etching. Langmuir. 20:4720-4727. http://dx.doi.org/10.1021/ la036219j 
Li, Z.F., and E. Ruckenstein. 2004. Water-soluble poly(acrylic acid) grafted luminescent silicon nanoparticles and their use as fluorescent biological staining labels. Nano Lett. 4:1463-1467 http://dx.doi.org/10 $.1021 / \mathrm{n} 10492436$

Li-Shishido, S., T.M. Watanabe, H. Tada, H. Higuchi, and N. Ohuchi. 2006. Reduction in nonfluorescence state of quantum dots on an immunofluorescence staining. Biochem. Biophys. Res. Commun. 351:7-13. http://dx .doi.org/10.1016/j.bbrc.2006.09.159

Liu, D.S., W.S. Phipps, K.H. Loh, M. Howarth, and A.Y. Ting. 2012. Quantum dot targeting with lipoic acid ligase and HaloTag for single-molecule imaging on living cells. ACS Nano. 6:11080-11087.

Mahler, B., P. Spinicelli, S. Buil, X. Quelin, J.P. Hermier, and B. Dubertret. 2008. Towards non-blinking colloidal quantum dots. Nat. Mater. 7:659 664. http://dx.doi.org/10.1038/nmat2222

McRae, S.R., C.L. Brown, and G.R. Bushell. 2005. Rapid purification of EGFP, EYFP, and ECFP with high yield and purity. Protein Expr. Purif. 41:121127. http://dx.doi.org/10.1016/j.pep.2004.12.030

Melhuish, W.H. 1961. Quantum efficiencies of fluorescence of organic substances: Effect of solvent and concentration of fluorescent solute. J. Phys. Chem. 65:229-235. http://dx.doi.org/10.1021/j100820a009

Mujumdar, R.B., L.A. Ernst, S.R. Mujumdar, C.J. Lewis, and A.S. Waggoner 1993. Cyanine dye labeling reagents: sulfoindocyanine succinimidyl esters Bioconjug. Chem. 4:105-111. http://dx.doi.org/10.1021/bc00020a001

Murakoshi, H., R. Iino, T. Kobayashi, T. Fujiwara, C. Ohshima, A. Yoshimura, and A. Kusumi. 2004. Single-molecule imaging analysis of Ras activation in living cells. Proc. Natl. Acad. Sci. USA. 101:7317-7322. http://dx.doi .org/10.1073/pnas.0401354101

Muro, E., T. Pons, N. Lequeux, A. Fragola, N. Sanson, Z. Lenkei, and B Dubertret. 2010. Small and stable sulfobetaine zwitterionic quantum dots for functional live-cell imaging. J. Am. Chem. Soc. 132:4556-4557. http://dx.doi.org/10.1021/ja1005493

Mutch, S.A., B.S. Fujimoto, C.L. Kuyper, J.S. Kuo, S.M. Bajjalieh, and D.T Chiu. 2007. Deconvolving single-molecule intensity distributions for quantitative microscopy measurements. Biophys. J. 92:2926-2943. http://dx.doi.org/10.1529/biophysj.106.101428

O'Connor, T.F., P.G. Debenedetti, and J.D. Carbeck. 2007. Stability of proteins in the presence of carbohydrates; experiments and modeling using scaled particle theory. Biophys. Chem. 127:51-63. http://dx.doi.org/10.1016/ j.bpc.2006.12.004

Rigler, R., Ü. Mets, J. Widengren, and P. Kask. 1993. Fluorescence correlation spectroscopy with high count rate and low background: Analysis of translational diffusion. Eur. Biophys. J. 22:169-175. http://dx.doi.org/ 10.1007/BF00185777

Simson, R., E.D. Sheets, and K. Jacobson. 1995. Detection of temporary lateral confinement of membrane proteins using single-particle tracking analysis. Biophys. J. 69:989-993. http://dx.doi.org/10.1016/S0006-3495 (95)79972-6

Smith, A.M., and S. Nie. 2010. Semiconductor nanocrystals: structure, properties, and band gap engineering. Acc. Chem. Res. 43:190-200. http:// dx.doi.org/10.1021/ar9001069

Sychugov, I., R. Juhasz, J. Valenta, and J. Linnros. 2005a. Narrow luminescence linewidth of a silicon quantum dot. Phys. Rev. Lett. 94:087405. http:// dx.doi.org/10.1103/PhysRevLett.94.087405

Sychugov, I., R. Juhasz, J. Linnros, and J. Valenta. 2005b. Luminescence blinking of a $\mathrm{Si}$ quantum dot in a $\mathrm{SiO}_{2}$ shell. Phys. Rev. B. 71:1153311-115331-5. http://dx.doi.org/10.1103/PhysRevB.71.115331

Thomas, J.C. 1987. The determination of log normal particle size distributions by dynamic light scattering. J. Colloid Interface Sci. 117:187-192. http:// dx.doi.org/10.1016/0021-9797(87)90182-2

Toomre, D., and J. Bewersdorf. 2010. A new wave of cellular imaging. Annu. Rev. Cell Dev. Biol. 26:285-314. http://dx.doi.org/10.1146/annurevcellbio-100109-104048

Torres, A.J., M. Wu, D. Holowka, and B. Baird. 2008. Nanobiotechnology and cell biology: micro- and nanofabricated surfaces to investigate receptor-mediated signaling. Аппи Rev Biophys. 37:265-288. http://dx.doi .org/10.1146/annurev.biophys.36.040306.132651

Umemura, Y.M., M. Vrljic, S.Y. Nishimura, T.K. Fujiwara, K.G.N. Suzuki, and A. Kusumi. 2008. Both MHC class II and its GPI-anchored form undergo hop diffusion as observed by single-molecule tracking. Biophys. J. 95:435-450. http://dx.doi.org/10.1529/biophysj.107.123018

van Sark, W.G.J.H.M., P.L.T.M. Frederix, A.A. Bol, H.C. Gerritsen, and A. Meijerink. 2002. Blueing, bleaching, and blinking of single CdSe/ZnS quantum dots. ChemPhysChem. 3:871-879. http://dx.doi.org/10.1002/14397641(20021018)3:10<871::AID-CPHC871>3.0.CO;2-T

Vogelsang, J., R. Kasper, C. Steinhauer, B. Person, M. Heilemann, M. Sauer, and P. Tinnefeld. 2008. A reducing and oxidizing system minimizes photobleaching and blinking of fluorescent dyes. Angew. Chem. Int. Ed. Engl. 47:5465-5469. http://dx.doi.org/10.1002/anie.200801518
Wang, L., V. Reipa, and J. Blasic. 2004. Silicon nanoparticles as a luminescent label to DNA. Bioconjug. Chem. 15:409-412. http://dx.doi.org/10.1021/ bc030047k

Wang, X., X. Ren, K. Kahen, M.A. Hahn, M. Rajeswaran, S. MaccagnanoZacher, J. Silcox, G.E. Cragg, A.L. Efros, and T.D. Krauss. 2009. Nonblinking semiconductor nanocrystals. Nature. 459:686-689. http://dx.doi .org/10.1038/nature08072

Warner, J.H., A. Hoshino, K. Yamamoto, and R.D. Tilley. 2005. Water-soluble photoluminescent silicon quantum dots. Angew. Chem. Int. Ed. Engl. 44:4550-4554. http://dx.doi.org/10.1002/anie.200501256

West, A.P., Jr., M.J. Bennett, V.M. Sellers, N.C. Andrews, C.A. Enns, and P.J. Bjorkman. 2000. Comparison of the interactions of transferrin receptor and transferrin receptor 2 with transferrin and the hereditary hemochromatosis protein HFE. J. Biol. Chem. 275:38135-38138. http://dx.doi .org/10.1074/jbc.C000664200

Yamani, Z., N. Rigakis, and M.H. Nayfeh. 1998. Excitation of size selected nanocrystallites in porous silicon. Appl. Phys. Lett. 72:2556-2558. http:// dx.doi.org/10.1063/1.121416

Yan, Y., M.E. Marriott, C. Petchprayoon, and G. Marriott. 2011. Optical switch probes and optical lock-in detection (OLID) imaging microscopy: highcontrast fluorescence imaging within living systems. Biochem. J. 433: 411-422. http://dx.doi.org/10.1042/BJ20100992

Yao, J., D.R. Larson, H.D. Vishwasrao, W.R. Zipfel, and W.W. Webb. 2005. Blinking and nonradiant dark fraction of water-soluble quantum dots in aqueous solution. Proc. Natl. Acad. Sci. USA. 102:14284-14289. http:// dx.doi.org/10.1073/pnas.0506523102

Yoshida, N., M. Kinjo, and M. Tamura. 2001. Microenvironment of endosomal aqueous phase investigated by the mobility of microparticles using fluorescence correlation spectroscopy. Biochem. Biophys. Res. Commun. 280:312-318. http://dx.doi.org/10.1006/bbrc.2000.4115

Zacharias, D.A., J.D. Violin, A.C. Newton, and R.Y. Tsien. 2002. Partitioning of lipid-modified monomeric GFPs into membrane microdomains of live cells. Science. 296:913-916. http://dx.doi.org/10.1126/science.1068539

Zhou, Z.Y., L. Brus, and R. Friesner. 2003. Electronic structure and luminescence of 1.1- and 1.4-nm silicon nanocrystals: Oxide shell versus hydrogen passivation. Nano Lett. 3:163-167. http://dx.doi.org/10.1021/n1025890q 